\title{
More on tie-points and homeomorphism in $\mathbb{N}^{*}$
}

\author{
by \\ Alan Dow (Charlotte, NC) and \\ Saharon Shelah (Piscataway, NJ, and Jerusalem)
}

\begin{abstract}
A point $x$ is a (bow) tie-point of a space $X$ if $X \backslash\{x\}$ can be partitioned into (relatively) clopen sets each with $x$ in its closure. We denote this as $X=A \bowtie B$ where $A, B$ are the closed sets which have a unique common accumulation point $x$. Tie-points have appeared in the construction of non-trivial autohomeomorphisms of $\beta \mathbb{N} \backslash \mathbb{N}=\mathbb{N}^{*}$ (by Veličković and Shelah \& Steprāns) and in the recent study (by Levy and Dow \& Techanie) of precisely 2-to- 1 maps on $\mathbb{N}^{*}$. In these cases the tie-points have been the unique fixed point of an involution on $\mathbb{N}^{*}$. One application of the results in this paper is the consistency of there being a 2-to-1 continuous image of $\mathbb{N}^{*}$ which is not a homeomorph of $\mathbb{N}^{*}$.
\end{abstract}

1. Introduction. A point $x$ is a tie-point of a space $X$ if there are closed sets $A, B$ of $X$ such that $X=A \cup B,\{x\}=A \cap B$ and $x$ is an adherent point of both $A$ and $B$. We let $X=A \bowtie B$ denote this relation and say that $x$ is a tie-point as witnessed by $A, \stackrel{x}{B}$. Let $A \equiv_{x} B$ mean that there is a homeomorphism from $A$ to $B$ with $x$ as a fixed point. If $X=A \bowtie B$ and $A \equiv_{x} B$, then there is an involution $\Phi$ of $X$ (i.e. $\Phi^{2}=\Phi$ ) such that $\{x\}=\operatorname{fix}(\Phi)$. In this case we will say that $x$ is a symmetric tie-point of $X$.

Let $\Phi$ be a continuous function from $\mathbb{N}^{*}$ into $\mathbb{N}^{*}$. Of course, $\Phi^{-1}$ can be regarded as a function from the clopen subsets of $\mathbb{N}^{*}$ into the clopen subsets of $\mathbb{N}^{*}$. A function $F$ from $\mathcal{P}(\mathbb{N})$ into $\mathcal{P}(\mathbb{N})$ is a lifting of $\Phi$ if $F(a)^{*}=$ $\Phi^{-1}\left(a^{*}\right)$ for all $a \subset \mathbb{N}$. A function $h$ is said to induce $F$ (and/or $\left.\Phi\right)$ on $I$ if $F(a)={ }^{*} h[a]=\{h(n): n \in a\}$ for all $a \subset I$. The function $F$ is said to be trivial on $I$ if there is such a function $h$. Since the fixed point set of a trivial autohomeomorphism is clopen, a symmetric tie-point gives rise to a non-trivial autohomeomorphism. An ideal on $\mathbb{N}$ is a $P$-ideal if it is countably directed closed mod finite.

2000 Mathematics Subject Classification: 03A50, 54A25, 54D35.

Key words and phrases: automorphism, Stone-Čech, fixed points.

This is paper number B917 in the second author's personal listing. 
If $A$ and $B$ are arbitrary compact spaces, and if $x \in A$ and $y \in B$ are accumulation points, then let $A \underset{x=y}{\bowtie} B$ denote the quotient space of $A \oplus B$ obtained by identifying $x$ and $y$, and let $x y$ denote the collapsed point. Clearly the point $x y$ is a tie-point of this space.

In this paper we establish the following theorem.

TheOREM 1.1. It is consistent that $\mathbb{N}^{*}$ has symmetric tie-points $x, y$ as witnessed by $A, B$ and $A^{\prime}, B^{\prime}$ respectively such that $\mathbb{N}^{*}$ is not homeomorphic to the space $A \underset{x=y}{\bowtie} A^{\prime}$.

COROLlary 1.2. It is consistent that there is a 2-to-1 image of $\mathbb{N}^{*}$ which is not a homeomorph of $\mathbb{N}^{*}$.

One can generalize the notion of tie-point and, for a point $x \in \mathbb{N}^{*}$, consider how many disjoint clopen subsets of $\mathbb{N}^{*} \backslash\{x\}$ (each accumulating to $x$ ) can be found. Let us say that a tie-point $x$ of $\mathbb{N}^{*}$ satisfies $\tau(x) \geq n$ if $\mathbb{N}^{*} \backslash\{x\}$ can be partitioned into $n$ disjoint clopen subsets each accumulating to $x$. Naturally, we will let $\tau(x)=n$ denote that $\tau(x) \geq n$ and $\tau(x) \nsupseteq n+1$. It follows easily from $[2,5.1]$ that each point $x$ of character $\omega_{1}$ in $\mathbb{N}^{*}$ is a tie-point and satisfies $\tau(x) \geq n$ for all $n$. Similarly each $P$-point of character $\omega_{1}$ in $\mathbb{N}^{*}$ is a symmetric tie-point. We list several open questions in the final section.

THeOREM 1.3. It is consistent that $\mathbb{N}^{*}$ has a tie-point $x$ such that $\tau(x)=2$ and $\mathbb{N}^{*}=A \underset{x}{\bowtie} B$, where neither $A$ nor $B$ is a homeomorph of $\mathbb{N}^{*}$. In addition, there are no symmetric tie-points.

The following theorem of [10] provides an important equivalent condition for the triviality of autohomeomorphisms on $\mathbb{N}^{*}$ and it will allow us to utilize the results of Steprāns's paper [8].

Lemma 1.4 (Veličković). If $F: \mathcal{P}(\mathbb{N}) \rightarrow \mathcal{P}(\mathbb{N})$ is a lifting of an autohomeomorphism and there exist Borel functions $\left\{\psi_{n}: n \in \omega\right\}$ and a comeager set $G \subset \mathcal{P}(\mathbb{N})$ such that for every $A \in G$ there is $n \in \omega$ such that $\psi_{n}(A)={ }^{*} F(A)$, then $F$ is trivial.

This is Theorem 2 of [10] except that the strengthening to the case of a comeager set $G$ is from $[8,2.1]$. The topology on $\mathcal{P}(\mathbb{N})$ is the standard one induced by identifying each set $a \subset \mathbb{N}$ with its characteristic function $\chi_{a} \in 2^{\mathbb{N}}$. For a set $\mathcal{C} \subset \mathcal{P}(\mathbb{N})$ and a function $F$ on $\mathcal{P}(\mathbb{N})$, let us say that $F\lceil\mathcal{C}$ is $\sigma$-Borel if there is sequence $\left\{\psi_{n}: n \in \omega\right\}$ of Borel functions on $\mathcal{P}(\mathbb{N})$ such that for each $b \in \mathcal{C}$, there is an $n$ such that $F(b)={ }^{*} \psi_{n}(b)$.

The following lemma is also implicit in $[10,1.3]$ :

LEMMA 1.5. If $F$ is a lifting of an autohomeomorphism of $\mathbb{N}^{*}$ and if $F$ is trivial on each member of a $P$-ideal $\mathcal{I}$ for which $F \nmid \mathcal{I}$ is $\sigma$-Borel, then there is a function $h$ which induces $F$ on each member of $\mathcal{I}$. 
The following partial order $\mathbb{P}_{2}$ was introduced by Veličković in [10]. Our need for this poset is articulated in [8] where it is described as a poset which was introduced "to add a non-trivial automorphism of $\mathcal{P}(\mathbb{N}) /[\mathbb{N}]<\aleph_{0}$ while doing as little else as possible - at least assuming PFA".

Definition 1.6. The partial order $\mathbb{P}_{2}$ is defined to consist of all 1-to-1 functions $f: A \rightarrow B$ where

- $A \subseteq \omega$ and $B \subseteq \omega \backslash A$,

- for all $i \in \omega$ and $n \in \omega, f(i) \in 2^{n+1} \backslash 2^{n}$ if and only if $i \in 2^{n+1} \backslash 2^{n}$,

- $\lim \sup _{n \rightarrow \omega}\left|\left(2^{n+1} \backslash 2^{n}\right) \backslash(A \cup B)\right|=\omega$.

The ordering on $\mathbb{P}_{2}$ is $\subseteq^{*}$.

We define some trivial generalizations of $\mathbb{P}_{2}$. We use the notation $\mathbb{P}_{2}$ to signify that this poset introduces an involution of $\mathbb{N}^{*}$ because the condition $g=f \cup f^{-1}$ implies that $g^{2}=g$. In the definition of $\mathbb{P}_{2}$ it is possible to suppress mention of $A, B$ (which we do) and to have the poset $\mathbb{P}_{2}$ consist simply of the functions $g$ (and $A$ as $L_{g}=\{i \in \operatorname{dom}(g): i<g(i)\}$, and $B$ as $\left.U_{g}=\{i \in \operatorname{dom}(g): g(i)<i\}\right)$.

Let $\mathbb{P}_{1}$ denote the poset we get if we omit mention of $f$ consisting only of disjoint pairs $(A, B)$, satisfying the growth condition in Definition 1.6, and extension is coordinatewise mod finite containment. For more consistent notation, we will instead represent the elements of $\mathbb{P}_{1}$ as partial functions into 2 .

More generally, let $\mathbb{P}_{l}$ be similar to $\mathbb{P}_{2}$ except that we assume that conditions consist of functions $g$ such that $\left\{i, g(i), g^{2}(i), \ldots, g^{l}(i)\right\}$ is contained in $l^{n+1} \backslash l^{n}$ and has precisely $l$ elements for all $i \in \operatorname{dom}(g) \cap l^{n+1} \backslash l^{n}$.

The basic properties of $\mathbb{P}_{2}$ as defined by Veličković and treated by Shelah and Steprāns are also true of $\mathbb{P}_{l}$ for all $l \in \mathbb{N}$.

In particular, for example, the following is easily seen:

Proposition 1.7. If $L \subset \mathbb{N}$ and $\mathbb{P}=\prod_{l \in L} \mathbb{P}_{l}$ (with full supports) and $G$ is a $\mathbb{P}$-generic filter, then in $V[G]$, for each $l \in L$, there is a tie-point $x_{l} \in \mathbb{N}^{*}$ with $\tau\left(x_{l}\right) \geq l$.

For the proof of Theorem 1.1 we use $\mathbb{P}_{2} \times \mathbb{P}_{2}$ and for the proof of Theorem 1.3 we use $\mathbb{P}_{1}$. In any such $\mathbb{P}$ and $\vec{f}, \vec{g} \in \mathbb{P}$, we say that $\vec{f}=\left\langle f_{l}\right\rangle$ is an $n$-preserving extension of $\vec{g}=\left\langle g_{l}\right\rangle$, for an integer $n$, if for each coordinate $l$, $f_{l}\left\lceil n=g_{l}\left\lceil n\right.\right.$ and $f_{l} \supset g_{l}$. Also, if $\vec{s}=\left\langle s_{l}\right\rangle$ is a sequence of functions (usually with finite domain), then we define $\vec{s} \sqcup \vec{f}$ to be the sequence $\vec{g}=\left\langle g_{l}\right\rangle$ where, for each coordinate $l$,

$$
g_{l}=s_{l} \sqcup f_{l} \equiv s_{l} \cup\left(f_{l}\left\lceil\operatorname{dom}\left(f_{l}\right) \backslash \operatorname{dom}\left(s_{l}\right)\right) .\right.
$$


2. Preliminaries. Each poset $\mathbb{P}$ as above is $\aleph_{1}$-closed and, if PFA holds, $\aleph_{2}$-distributive (see $[8$, p. 4226$]$ ). In this paper we will restrict our study to finite products. The following partial order can be used to show that these products are $\aleph_{2}$-distributive.

Definition 2.1. Let $\mathbb{P}$ be a finite product of posets from $\left\{\mathbb{P}_{l}: l \in \mathbb{N}\right\}$. Given $\mathfrak{F} \subset \mathbb{P}$, define $\mathbb{P}(\mathfrak{F})$ to be the partial order consisting of all $g \in \mathbb{P}$ such that there is some $\vec{f} \in \mathfrak{F}$ such that $\vec{g} \equiv^{*} \vec{f}$. The ordering on $\mathbb{P}(\mathfrak{F})$ is coordinatewise $\supseteq$ as opposed to ${ }^{*} \supseteq$ in $\mathbb{P}$.

If $\mathfrak{F}$ is downward directed (in fact it will be a descending sequence), then the forcing $\mathbb{P}(\mathfrak{F})$ introduces a tuple $\vec{f}$ such that $\vec{f} \leq \vec{f}^{\prime}$ for all $\vec{f}^{\prime} \in \mathfrak{F}$. Although $\vec{f}$ itself may not be a member of $\mathbb{P}$, it is simply because the domains of the component functions are too big. Following [6, 2.1], one must then use a $\sigma$-centered poset which will choose an appropriate sequence $\vec{f}^{*}$ of subfunctions of $\vec{f}$ which is a member of $\mathbb{P}$ and which is still below each member of $\mathfrak{F}$.

A strategic choice of the sequence $\mathfrak{F}$ will ensure that $\mathbb{P}(\mathfrak{F})$ is ccc, but remarkably even more is true. Again we are lifting results from $[6,2.6]$ and $[8$, proof of Thm. 3.1] which introduced this innovative factoring of Veličković's original amoeba forcing poset and showed that it seems to preserve more properties. Let $\omega_{2}^{<\omega_{1}}$ denote the standard collapse which introduces a function from $\omega_{1}$ onto $\omega_{2}$.

A poset is said to be $\omega^{\omega}$-bounding if every new function in $\omega^{\omega}$ is bounded by some ground model function.

Lemma 2.2. Let $\mathbb{P}$ be a finite product of posets from $\left\{\mathbb{P}_{l}: l \in \mathbb{N}\right\}$. In the forcing extension, $V[H]$, by $\omega_{2}^{<\omega_{1}}$, there is a descending sequence $\mathfrak{F}$ from $\mathbb{P}$ which is $\mathbb{P}$-generic over $V$ and for which $\mathbb{P}(\mathfrak{F})$ is ccc and $\omega^{\omega}$-bounding.

It was also shown in $[6]$ that $\mathfrak{F}$ can be chosen so that it, in addition, preserves that $\mathbb{R} \cap V$ is of second category. This is crucial for the proof of Lemma 2.3. We can manage with the $\omega^{\omega}$-bounding property because we are going to use Lemma 2.3. An ideal $\mathcal{I} \subset \mathcal{P}(\mathbb{N})$ is said to be dense if each infinite subset of $\mathbb{N}$ contains a member of $\mathcal{I}$.

The following main result is extracted from [6] and [8, Theorem 3.3] which we record without proof.

Lemma 2.3 (PFA). Let $\mathbb{P}$ be a finite product of posets from the set $\left\{\mathbb{P}_{l}\right.$ : $l \in \mathbb{N}\}$. Let $\dot{F}$ be a $\mathbb{P}$-name of a lifting of an autohomeomorphism of $\mathbb{N}^{*}$. Let $\mathfrak{F}$ and $H$ be as in Lemma 2.2 and let $F$ be the valuation of $\dot{F}$ by $\mathfrak{F}$. Then $F$ is a lifting of an autohomeomorphism of $\mathbb{N}^{*}($ in $V[H])$ and for any dense $P$-ideal $\mathcal{I}$ on $\mathbb{N}$ and for each $\mathbb{P}(\mathfrak{F})$-generic filter $G$, there is an $I \in \mathcal{I}$ such that $F \uparrow\left(V[H] \cap[\mathbb{N} \backslash I]^{\omega}\right)$ is $\sigma$-Borel in the extension $V[H][G]$. 
Let $\mathfrak{F}, \dot{F}, H$ and $F$ be as in Lemma 2.3 and consider the situation in the forcing extension $V[H]$. Since $\mathfrak{F}$ is generic over $V$, we will see that for each $Y \in[\mathbb{N}]^{\omega}$, there is some $f \in \mathfrak{F}$ which decides if $\dot{F}\left\lceil[Y]^{\omega}\right.$ is trivial. Also, the genericity of $\mathfrak{F}$ over $V$, and the fact that no new subsets of $\mathbb{N}$ are added, will ensure that if some $f$ in $\mathfrak{F}$ forces that $\dot{F} \uparrow[Y]^{\omega}$ is not trivial, then $F \uparrow[Y]^{\omega}$ is also not trivial (in $V[H]$ ). We will assume all these properties of $\mathfrak{F}$ and $F$ throughout the paper.

The following proposition is probably well-known but we do not have a reference.

Proposition 2.4. Assume that $\mathbb{Q}$ is a ccc $\omega^{\omega}$-bounding poset and that $x$ is an ultrafilter on $\mathbb{N}$. If $G$ is a $\mathbb{Q}$-generic filter then there is no set $A \subset \mathbb{N}$ such that $A \backslash Y$ is finite for all $Y \in x$.

Proof. Assume that $\left\{\dot{a}_{n}: n \in \omega\right\}$ are $\mathbb{Q}$-names of integers such that $1 \Vdash_{\mathbb{Q}}$ " $\dot{a}_{n} \geq n$ ". Let $A$ denote the $\mathbb{Q}$-name such that $\Vdash_{\mathbb{Q}} " A=\left\{\dot{a}_{n}: n \in \omega\right\} "$. Since $\mathbb{Q}$ is $\omega^{\omega}$-bounding, there is some $q \in \mathbb{Q}$ and a sequence $\left\{n_{k}: k \in \omega\right\}$ in $V$ such that $q \Vdash_{\mathbb{Q}} " n_{k} \leq \dot{a}_{i} \leq n_{k+2} \forall i \in\left[n_{k}, n_{k+1}\right)$ ". There is some $l \in 3$ such that $Y=\bigcup_{k}\left[n_{3 k+l}, n_{3 k+l+1}\right)$ is a member of $x$. On the other hand, for each $k, q \Vdash_{\mathbb{Q}}$ " $A \cap\left[n_{3 k+l+1}, n_{3 k+l+3}\right)$ is not empty". Therefore $q \nVdash_{\mathbb{Q}}$ " $A \backslash Y$ is finite".

Another interesting and useful general lemma is the following.

Lemma 2.5. Let $\mathbb{P}$ be a finite product of posets from the set $\left\{\mathbb{P}_{l}: l \in \mathbb{N}\right\}$. Let $H$ and $\mathfrak{F}$ be as in Lemma 2.2. Then for each $\mathbb{P}(\mathfrak{F})$-name $\dot{h} \in \mathbb{N}^{\mathbb{N}}$ there are an increasing sequence $n_{0}<n_{1}<\cdots$ of integers and a condition $\vec{f} \in \mathfrak{F}$ such that either

(1) $\vec{f} \Vdash_{\mathbb{P}(\mathfrak{F})}$ " $h \uparrow \bigcup\left\{\left[n_{k}, n_{k+1}\right): k \in K\right\} \notin V$ " for each infinite $K \subset \omega$, or

(2) for each $i \in\left[n_{k}, n_{k+1}\right)$ and each $\vec{g}<\vec{f}$ such that $\vec{g}$ forces a value on $\dot{h}(i),\left\langle f_{l} \cup\left(g_{l} \uparrow\left[n_{k}, n_{k+1}\right)\right)\right\rangle$ also forces a value on $\dot{h}(i)$.

Furthermore, if $\vec{f}$ forces $\dot{h}$ to be finite-to-one, we can arrange that for each $k$ and each $i \in\left[n_{k}, n_{k+1}\right), \vec{f}$ forces that $\dot{h}(i) \in\left[n_{k-1}, n_{k+2}\right)$.

Proof. Fix any $\vec{f} \in \mathbb{P}$. Perform a standard fusion, as in $[6,2.4]$ or $[8,3.4]$, to find sequences $\left\{n_{k}: k \in \omega\right\} \subset \omega$ and $\left\{\vec{f}^{k}: k \in \omega\right\} \subset \mathbb{P}$ with the following properties. Each $\vec{f}^{k}$ is an $n_{k}$-preserving extension of $\vec{f}^{k-1}$. Let $j<n_{k}$ and let $\vec{s}, \vec{s}^{*} \in \mathbb{P}$ be such that, for each coordinate $l$ of $\mathbb{P}, s_{l} \subset s_{l}^{*}$ and $s_{l}$ has domain contained in $n_{k}$. If there is some $n_{k}$-preserving extension of $\vec{s} \sqcup \vec{f}^{k}$ which forces a value on $\dot{h}(j)$, then $\vec{s} \sqcup \vec{f}^{k}$ already does so. Further, if there is some integer $i \geq n_{k}$ for which $\vec{s}^{*} \sqcup \vec{f}^{k}$ has an $n_{k}$-preserving extension forcing a value on $\dot{h}(i)$ while $\vec{s} \sqcup \vec{f}^{k}$ does not, then there is such an integer below $n_{k+1}$. One also ensures that for each coordinate $l$ there is an $m$ such that 
$n_{k}<2^{m}<2^{m+1}<n_{k+1},\left[2^{m}, 2^{m+1}\right) \backslash \operatorname{dom}\left(f_{l}^{k}\right)$ has at least $k$ elements (thus ensuring that the end result of the fusion will be a member of $\mathbb{P}$ ).

Let $\overrightarrow{f^{\prime}}$ be the fusion and assume that $\underline{f}<\vec{f}^{\prime}$ and $K \in[\omega]^{\omega}$ are such that $\underline{f}$ forces a value on $\dot{h} \uparrow\left[n_{k}, n_{k+1}\right)$ for all $\vec{k} \in K$. We show that the second alternative then holds. By further extending $\underline{f}$ we can assume that if $L=$ $\left\{m:(\exists l)\left[n_{m}, n_{m+1}\right) \not \subset \operatorname{dom}\left(f_{l}\right)\right\}$, then $K \cap\left[m, m^{\prime}\right]$ is not empty for all $m<m^{\prime} \in L$.

Let $i \in\left[n_{m^{\prime}}, n_{m^{\prime}+1}\right)$ and let $\vec{g}<\underline{f}$ force a value on $\dot{h}(i)$. Assume that $\left\langle g_{l}\left\lceil\left[n_{m^{\prime}}, n_{m^{\prime}+1}\right)\right\rangle \sqcup \underline{f}\right.$ does not force a value on $\dot{h}(i)$, and so has no $n_{m^{\prime}+1^{-}}$ preserving extension which does.

Let $m$ be the maximum member of $L \cap m^{\prime}$ and choose $k \in K \cap\left[m, m^{\prime}\right]$. Set $\vec{s}=\left\langle f_{l}^{\prime}\left\lceil n_{k}\right\rangle\right.$ and $\vec{s}^{\prime}=\left\langle\vec{g}_{l}\left\lceil n_{k}\right\rangle\right.$. We note that $i$ is a witness to the situation that $\vec{s}^{\prime} \sqcup \vec{f}^{n_{k}}$ has an $n_{k}$-preserving extension to decide, while $\vec{s} \sqcup \vec{f}^{n_{k}}$ does not. Therefore, by construction, there should be some $j<n_{k+1}$ for which this is true. However, this is not the case since $\underline{f}$ forces a value on $\dot{h} \uparrow\left[n_{k}, n_{k+1}\right)$.

If $\vec{f}$ forces that $\dot{h}$ is finite-to-one, then $\vec{f}^{0}$ could have been so chosen. In addition, since $\mathbb{P}(\mathfrak{F})$ is $\omega^{\omega}$-bounding we may fix an increasing function $g \in \omega^{\omega} \cap V$ such that (if $\vec{f}$ forces $\dot{h}$ is finite-to-one) $\vec{f} \Vdash_{\mathbb{P}(\mathfrak{F})}$ " $\{i, \dot{h}(i)\} \cup$ $\dot{h}^{-1}(i) \subset g(i)$ ". The only change to the fusion is to additionally demand that $n_{k+1}$ is chosen to be larger than $g\left(n_{k}\right)$ at each stage.

3. The trivial ideal. In this section we establish a result that will guarantee that our autohomeomorphisms of $\mathbb{N}^{*}$ will be trivial on every member of a large $P$-ideal.

Lemma 3.1. Let $\mathbb{P}$ be a finite product of posets from the set $\left\{\mathbb{P}_{l}: l \in \mathbb{N}\right\}$, let $H$ and $\mathfrak{F}$ be as in Lemma 2.2 , and let $G$ be $\mathbb{P}(\mathfrak{F})$-generic over $V[H]$. Assume that $b \in V \cap[\mathbb{N}]^{\omega}$ is such that $F \uparrow\left[V \cap[b]^{\omega}\right]$ is $\sigma$-Borel in $V[G]$. Then, in $V$, there is an increasing sequence $\left\{n_{k}: k \in \omega\right\} \subset \omega$ such that $F$ is trivial on each $a \in[b]^{\omega}$ for which there is an $r \in \mathfrak{F}$ such that $a \subset \bigcup\left\{\left[n_{k}, n_{k+1}\right)\right.$ : $\left.\left[n_{k}, n_{k+1}\right) \subset \operatorname{dom}(r)\right\}$.

Proof. For notational convenience we will assume that $\mathbb{P}$ is simply a single member of $\left\{\mathbb{P}_{l}: l \in \mathbb{N}\right\}$. The modifications needed to handle a finite product are completely straightforward and will be omitted.

Fix names $\dot{\psi}_{j}(j \in \omega)$ for the Borel functions. Fix an appropriately large countable elementary submodel $M \prec H(\theta)$. For easier notation, we may just assume that $b$ is actually $\mathbb{N}$. We will use the notation $p$ with subscripts to refer to members of $\mathbb{P}$. For a finite set $t \subset \mathbb{N}$ and $n \in \mathbb{N}$, we will use $[t ; n]$ to denote the clopen set $\{a \subset \mathbb{N}: a \cap n=t\}$. 
We first want to show that we can assume that each $\dot{\psi}_{j}$ is actually continuous. As is well-known, each Borel function is continuous on a dense $G_{\delta}$, hence we may fix a sequence $\left\{\dot{U}_{n}: n \in \omega\right\}$ of $\mathbb{P}(\mathfrak{F})$-names of a descending sequence of dense open sets such that each $\dot{\psi}_{j}$ is forced to be continuous on the intersection $\bigcap_{n} \dot{U}_{n}$. We perform a fusion sequence $\left\{p_{k}: k \in \omega\right\}$ (as in Lemma 3.1) which selects a sequence of intervals $\left\{\left[n_{k}, n_{k+1}\right): k \in \omega\right\}$, and finite sets $t_{k}$ contained in $\left[n_{k}, n_{k+1}\right.$ ), so that (it is forced by $p_{k}$ that) for each $s \subset n_{k},\left[s \cup t_{k} ; n_{k+1}\right]$ is a subset of $\dot{U}_{k}$. We deal with $F \nmid V \cap\left[\bigcup_{k}\left[n_{2 k}, n_{2 k+1}\right)\right]^{\omega}$ (and by symmetry) with $F \uparrow V \cap\left[\bigcup_{k}\left[n_{2 k-1}, n_{2 k}\right)\right]^{\omega}$ ) by replacing, for $y \subset$ $\bigcup_{k}\left[n_{2 k}, n_{2 k+1}\right), \dot{\psi}_{j}(y)$ with $\dot{\psi}_{j}\left(y \cup \bigcup_{k} t_{2 k}\right) \backslash F\left(\bigcup_{k} t_{2 k}\right)$. Thus, we may simply assume that each $\dot{\psi}_{j}$ is continuous.

We perform another fusion sequence and produce a new sequence $\left\{n_{i}\right.$ : $i \in \omega\}$. This also is all done in $M$. For each $i$ we will select a subset $f_{i} \subset\left[n_{i}, n_{i+1}\right)$ and we are trying to imitate the "forcing a value" idea from [9]. That is, for each $i$ and each $s \in n_{i}^{n_{i}}$ and $t \subset n_{i}$ and each $j \leq i$, we arrange that if $s \sqcup p_{i}$ has an $n_{i}$-preserving extension which is able to force a value on $\dot{\psi}_{j}\left[t \cup f_{i} ; n_{i+1}\right]\left\lceil n_{i}\right.$ (meaning all $a \in \dot{\psi}_{j}\left[t \cup f_{i} ; n_{i+1}\right]$ have the same intersection with $n_{i}$ ), then we do so (i.e. by possibly extending $f_{i}$ or by extending $\left.p_{i} \uparrow\left[n_{i}, \infty\right)\right)$. An additional requirement is to further finitely extend $f_{i}$, if possible, so that instead, there is some integer $m$ (which will be made to be less than $n_{i+1}$ ) so that $s \sqcup p_{i}$ has no $n_{i^{-}}$ preserving extension and $f_{i} ; n_{i}$ has no further finite extension $h ; n^{\prime}$, which will force a value on $\dot{\psi}_{j}\left[t \cup h ; n^{\prime}\right]\lceil m$. As usual, we also ensure that for each $i$, there is a unique $m_{i}$ such that $\left[2^{m_{i}}, 2^{m_{i}+1}\right) \subset\left[n_{i}, n_{i+1}\right)$ and $\operatorname{dom}\left(p_{i}\right) \supset$ $\left[n_{i}, n_{i+1}\right) \backslash\left[2^{m_{i}}, 2^{m_{i}+1}\right)$ and $\left|\left[2^{m_{i}}, 2^{m_{i}+1}\right) \backslash \operatorname{dom}\left(p_{i}\right)\right| \geq i$.

For $e \in\{0,1,2\}$, let $f^{e}=\bigcup_{i} f_{3 i+e}$. Choose a $p \in M$ such that $p$ decides the value of $F\left(f^{e}\right)$ for each such $e$. We will focus on $f^{0}$ but the following argument can be repeated for $f^{1}$ and $f^{2}$.

We perform another fusion choosing $\left\{i_{l}: l \in \omega\right\} \subset\{3 i: i \in \omega\}$ and conditions $r_{l}$. Again, with $n=n_{i_{l}}$, for each $j \leq i_{l}, s \in n^{n}$, and $t \subset n$, we choose $r=r_{l}$ to be an $n$-preserving extension so that either $s \sqcup r$ has forced a value on $\dot{\psi}_{j}\left(t \cup f^{0}\right)$, or there is a $3 i<i_{l+1}$ such that $s \sqcup r$ has no $n$-preserving extension which forces a value on $\dot{\psi}_{j}\left[\left(t \cup f^{0}\right) \cap n_{3 i+1} ; n_{3 i+1}\right]\left\lceil n_{3 i}\right.$.

Let $r<p$ extend this final fusion sequence and be an $(M, \mathbb{P})$-generic condition. For each $s$, let $s \sqcup r \in G_{s}$ be some $\mathbb{P}(\mathfrak{F})$-filter which is generic over $M$. This gives us a countable family of Borel functions $\left\{\operatorname{val}_{G_{s}}\left(\dot{\psi}_{j}\right)\right.$ : $\left.s \in \omega^{<\omega}, j \in \omega\right\}$ in $V[H]$ (and in $V$ ).

Let $L \subset \omega$ be any set of integers such that $\left[n_{i_{l}}, n_{i_{l+1}}\right) \subset \operatorname{dom}(r)$ for $l \in L$ and $L \cap\{l+1: l \in L\}$ is empty. Let $Y \subset \bigcup_{l \in L}\left[n_{i_{l}}, n_{i_{l+1}}\right)$ be such that, in addition, $Y \cap\left[n_{3 i}, n_{3 i+1}\right.$ ) (since we are using $f^{0}$ ) is empty for all $i$. To show that $F$ is trivial (using Proposition 1.4) on $[Y]^{\omega}$, we prove that for each 
$y \subset Y$, there are $s, j$ such that

$$
s \sqcup r \Vdash_{\mathbb{P}(\mathfrak{F})} \text { " } F(y)={ }^{*} \psi_{j}\left(y \cup f^{0}\right) \backslash F\left(f^{0}\right) " .
$$

It then follows, since $F(y)$ is an element of $V$, that $F(y)=\operatorname{val}_{G_{s}}\left(\dot{\psi}_{j}\right)\left(y \cup f^{0}\right)$ $\backslash F\left(f^{0}\right)$. Since all this is taking place in $V[H]$ we find that $F \uparrow[Y]^{\omega}$ is trivial.

Fix any $r_{y}<r$ which forces a value on $\dot{F}\left(y \cup f^{0}\right)$ and forces that this is equal to $\dot{\psi}_{j}\left(y \cup f^{0}\right)$ for some $j \in \omega$. Fix any $l_{0} \in L$ such that $j<i_{l_{0}}$ and set $s=r_{y}\left\lceil n_{i_{l_{0}}}\right.$. More generally, for each $l \in L$, let $s_{l}=r_{y}\left\lceil n_{i_{l}}\right.$. The next three claims complete the proof that $s=s_{0}$ and $j$ are as needed above.

Claim 1. For each $l \in \omega \backslash\left(L \cup l_{0}\right), s_{l} \sqcup r$ decides $\dot{\psi}_{j}\left(\left(y \cap n_{i_{l}}\right) \cup f^{0}\right)$.

Proof. Let $t=\left(y \cup f^{0}\right) \cap n_{i_{l}}$. Note also that $\left(y \cup f^{0}\right) \cap n_{i_{l+1}}$ is equal to $\left(t \cup f^{0}\right) \cap n_{i_{l+1}}$ since $l \notin L$. By assumption and continuity of $\dot{\psi}_{j}, r_{y}$ forces a value on $\dot{\psi}_{j}\left[\left(y \cup f^{0}\right) \cap n_{i_{l+1}} ; n_{i_{l+1}}\right]\left\lceil n_{3 i+1}\right.$ for each $3 i<i_{l+1}$. Therefore, $s_{l} \sqcup r_{l}$ did (does) have such an $n_{i_{l}}$-preserving extension to force values on $\dot{\psi}_{j}\left[\left(t \cup f^{0}\right) \cap n_{3 i+1} ; n_{3 i+1}\right]\left\lceil n_{3 i}\right.$ for each $3 i<i_{l+1}$. From this, it follows from the choice of $r_{l}$ that $s_{l} \sqcup r$ does force a value on $\dot{\psi}_{j}\left(t \cup f^{0}\right)$.

Claim 2. For each $l \in \omega \backslash l_{0}, s_{l} \sqcup r$ decides $\dot{\psi}_{j}\left(\left(y \cap n_{i_{l}}\right) \cup f^{0}\right)$.

Proof. By Claim 1, we may assume that $l \in L$ and so $l-1 \notin L$. We know by Claim 1 that $s_{l-1} \sqcup r$ decides $\dot{\psi}_{j}\left(\left(y \cap n_{i_{l}-1}\right) \cup f^{0}\right)$. But since $y \cap n_{i_{l}}$ is the same as $y \cap n_{i_{l-1}}$, it follows that $s_{l} \sqcup r$ decides $\dot{\psi}_{j}\left(\left(y \cap n_{i_{l}}\right) \cup f^{0}\right)$ since $s_{l-1} \sqcup r$ decides it.

Claim 3. For each $l \leq l^{\prime} \in \omega \backslash l_{0}, s_{l} \sqcup r$ decides $\dot{\psi}_{j}\left(\left(y \cap n_{i_{l^{\prime}}}\right) \cup f^{0}\right)$.

Proof. We proceed by induction on $l^{\prime}$. Assume the claim holds for $l^{\prime}$ and fails for $l^{\prime}+1$. Let $l$ be maximal such that it fails for $s_{l}$. We know that $l \leq l^{\prime}$ by Claim 2. It follows that we may assume that $t=y \cap n_{i_{l^{\prime}+1}} \neq y \cap n_{i_{l^{\prime}}}=t^{\prime}$, hence $l \in L$. When $r_{l^{\prime}+1}$ was defined, it was asked if $\left(\left(s_{l} \sqcup r\right)\left\lceil n_{i}\right) \sqcup r_{l^{\prime}+1}\right.$ had an $n_{i}$-preserving extension which forced a value on $\psi_{j}\left(t \cup f^{0}\right)$. Apparently the answer was no. But then, at stage $i=i_{l}$ in the $\left\langle p_{i}: i \in \omega\right\rangle$ fusion, it was asked if $t^{\prime} \cup f_{i}$ had an extension for which $\left(\left(s_{l} \cup r\right)\left\lceil n_{i}\right) \sqcup p_{i}\right.$ did not have an $n_{i}$-preserving extension to decide arbitrarily far. Well it appears that $t \cup f^{0}$ is such an extension (note that $f_{i} \subset f^{0}$ ). In this case, $f_{i} ; n_{i}$ were chosen so that it has no extension $h ; n^{\prime}$ for which $\left(\left(s_{l} \cup r\right)\left\lceil n_{i}\right) \sqcup p_{i}\right.$ has an $n_{i}$-preserving extension which will decide $\psi\left[t^{\prime} \cup h ; n^{\prime}\right]\left\lceil n_{i+1}\right.$. But we do know that $s_{l} \sqcup r$ decides $\dot{\psi}_{j}\left(t^{\prime} \cup f^{0}\right)=\dot{\psi}_{j}\left(\left(y \cap n_{i_{l^{\prime}}}\right) \cup f^{0}\right)$. Therefore at stage $i+3,\left(s_{l} \sqcup r\right)\left\lceil n_{i+3}\right.$ would have an $n_{i+2}$-preserving extension forcing a value on $\dot{\psi}_{j}\left[t \cup f_{i} \cup f_{i+3} ; n_{i+3}\right]\left\lceil n_{i+2}\right.$ (in fact, it would already do so). In particular, $t \cup f_{i} ; n_{i+1}$ does have an extension, namely $t \cup f_{i} \cup f_{i+3} ; n_{i+4}$, for which $\left(s_{l} \sqcup r\right)\left\lceil n_{i} \cup p_{i}\right.$ does have an $n_{i}$-preserving extension forcing a value on $\dot{\psi}_{j}\left[t \cup f_{i} \cup f_{i+3} ; n_{i+4}\right]\left\lceil n_{i+1}\right.$. 
This ends the proof of Lemma 3.1.

Corollary 3.2 (PFA). Let $\mathbb{P}$ be a finite product of posets from the set $\left\{\mathbb{P}_{l}: l \in \mathbb{N}\right\}$. Let $\dot{F}$ be a $\mathbb{P}$-name of a lifting of an autohomeomorphism of $\mathbb{N}^{*}$. Let $H, \mathfrak{F}, F, P$-ideal $\mathcal{I}$ and $I \in \mathcal{I}$ be as in Lemma 2.2. Then there is an increasing sequence $\left\{n_{k}: k \in \omega\right\} \subset \mathbb{N}$ and $a \mathbb{P}(\mathfrak{F})$-name $\dot{h}$ for a function on $\mathbb{N}$ such that for each $f \in \mathfrak{F}$ and $a=\bigcup\left\{\left[n_{k}, n_{k+1}\right):\left[n_{k}, n_{k+1}\right) \subset \operatorname{dom}(f)\right\}$, $F$ is trivial on $a \backslash I$ and $\mathbb{P}(\mathfrak{F})$ forces that $h \uparrow(a \backslash I)$ induces $F$.

Proof. Let $\left\{n_{k}: k \in \omega\right\}$ be the sequence as constructed in Lemma 3.1. Let $\mathcal{J}$ denote the dense $P$-ideal consisting of all sets of the form $\bigcup\left\{\left[n_{k}, n_{k+1}\right)\right.$ : $\left.\left[n_{k}, n_{k+1}\right) \subset \operatorname{dom}(f)\right\}$ for some $f \in \mathfrak{F}$. Since there is a natural (and obvious) finite-to-one map sending the ideal $\mathcal{J}$ to an ultrafilter, it follows by Proposition 2.4 that $\mathcal{J}$ generates a dense $P$-ideal in the forcing extension by $\mathbb{P}(\mathfrak{F})$. By Lemma 2.2, we know that $F \uparrow\left(V[H] \cap[\mathbb{N} \backslash I]^{\omega}\right)$ is $\sigma$-Borel. Let $\mathcal{J}^{I}$ be the ideal $\{J \backslash I: J \in \mathcal{J}\}$. By Lemma 3.1, $F$ is trivial on $J$ for each $J \in \mathcal{J}^{I}$. It then follows easily that, in the forcing extension by $\mathbb{P}(\mathfrak{F}), F\left\lceil\mathcal{J}^{I}\right.$ is also $\sigma$-Borel. Finally, by Lemma 1.5 , there is an $\dot{h}$ as required.

\section{Proof of Theorem 1.1}

TheOREM 4.1 (PFA). In the forcing extension by $\mathbb{P}=\mathbb{P}_{2} \times \mathbb{P}_{2}$, there are symmetric tie-points $x, y$ as witnessed by $A, B$ and $C, D$ respectively such that $\mathbb{N}^{*}$ is not homeomorphic to the space $A \underset{x=y}{\bowtie} C$.

We briefly work in the forcing extension in order to select appropriate names. Let $G \subset \mathbb{P}_{2} \times \mathbb{P}_{2}$ be a generic filter. The tie-point $x$ as witnessed by $A, B$ will be the one given canonically by the $\mathbb{P}_{2}$-generic filter consisting of the first coodinates of $G$ (as per the notation following Definition 1.6). The tie-point $y$ as witnessed by $C, D$ will be given analogously by the second coordinates. More precisely the closed set $A$ will be the closure of the union of the collection $\left\{L_{f}^{*}:(\exists g)(f, g) \in G\right\}$, while $B$ will be the closure of the union of the collection $\left\{U_{f}^{*}:(\exists g)(f, g) \in G\right\}$. Of course, $x$ is the ultrafilter (a $P_{\omega_{2}}$-point) generated by the collection $\{\mathbb{N} \backslash \operatorname{dom}(f):(\exists g)(f, g) \in G\}$.

Fix any enumeration $\left\{a_{\alpha}: \alpha \in \omega_{2}\right\}$ of a mod finite increasing cofinal chain in $\left\{L_{f}:(\exists g)(f, g) \in G\right\}$ and similarly $\left\{c_{\alpha}: \alpha \in \omega_{2}\right\}$ for $\left\{L_{g}:(\exists f)\right.$ $(f, g) \in G\}$. We may represent $A \underset{x=y}{\bowtie} C$ as a quotient of $(\mathbb{N} \times 2)^{*}$ in which, for each $\alpha \in \omega_{2},\left(a_{\alpha} \times\{0\}\right)^{*} \cup\left(c_{\alpha} \times\{1\}\right)^{*}$ is mapped canonically to $a_{\alpha}^{*} \cup c_{\alpha}^{*}$ and the rest of the $(\mathbb{N} \times 2)^{*}$ is collapsed to a point. Assume there is a homeomorphism from this quotient space to $\mathbb{N}^{*}$ and let $F$ be any lifting, i.e. we may assume that $F$ is a function from $[\mathbb{N}]^{\omega}$ into $[\mathbb{N} \times 2]^{\omega}$ such that if we let $Z_{\alpha}=F^{-1}\left(a_{\alpha} \times\{0\} \cup c_{\alpha} \times\{1\}\right)$ for each $\alpha \in \omega_{2}$, then $\left\{Z_{\alpha}: \alpha \in \omega_{2}\right\}$ forms the dual ideal $\mathcal{I}$ to an ultrafilter $z$. 
Fix $\mathbb{P}$-names for all the above mentioned objects and apply Corollary 3.2 to find the filter $\mathfrak{F} \subset \mathbb{P}$, the $\mathbb{P}$-name $\dot{h}$ and the sequence $\left\{n_{k}: k \in \omega\right\}$. There is no loss of generality in this proof to assume that the $I$ mentioned in the statement of Corollary 3.2 is the empty set, and let $\mathcal{J}$ be the ideal as defined in the proof of Corollary 3.2. As we are working in $V[H]$, let us use $\lambda$ to denote the $\omega_{2}$ from $V$. For each $J \in \mathcal{J}$, there is a function $h_{J}$ which induces $F$ on $J ; h_{J}$ will be a function from $J$ into $\left(a_{\alpha} \times\{0\} \cup\left(c_{\alpha} \times\{1\}\right)\right.$ for some $\alpha \in \lambda$.

We finish the proof by showing there is no such $\dot{h}$.

Since $\mathbb{P}(\mathfrak{F})$ is $\omega^{\omega}$-bounding, we may assume (by selecting a subsequence and renumbering) that the sequence $\left\{n_{k}: k \in \omega\right\}$ and some $\vec{f}_{0}=\left(g_{0}, g_{1}\right) \in \mathfrak{F}$ satisfy:

(1) for each $i \in\left[n_{k}, n_{k+1}\right), \vec{f}_{0} \Vdash_{\mathbb{P}(\mathfrak{F})}$ " $\dot{h}_{(i)} \in\left(\left[0, n_{k+2}\right) \times 2\right)$ ",

(2) for each $i \in\left[n_{k}, n_{k+1}\right), \overrightarrow{f_{0}} \Vdash_{\mathbb{P}(\mathfrak{F})}$ " $\dot{h}^{-1}(\{i\} \times 2) \subset\left[0, n_{k+2}\right)$ ",

(3) for each $k$ and each $j \in\{0,1\}$ there is an $m$ such that $n_{k}<2^{m}<$ $2^{m+1}<n_{k+1}$, and $\left[2^{m}, 2^{m+1}\right) \backslash \operatorname{dom} g_{j}$ has at least $k$ elements.

Choose any $\left(g_{0}^{\prime}, g_{1}^{\prime}\right)=\vec{f}_{1}<\vec{f}_{0}$ in $\mathfrak{F}$ such that $\mathbb{N} \backslash \operatorname{dom}\left(g_{0}^{\prime}\right)$ is contained in $\bigcup_{k}\left[n_{6 k+1}, n_{6 k+2}\right)$ and $\mathbb{N} \backslash \operatorname{dom}\left(g_{1}^{\prime}\right) \subset \bigcup_{k}\left[n_{6 k+4}, n_{6 k+5}\right)$. Next, choose any $\overrightarrow{f_{2}}<\overrightarrow{f_{1}}$ in $\mathfrak{F}$ and some $\alpha \in \lambda$ such that $\overrightarrow{f_{2}} \Vdash_{\mathbb{P}(\mathfrak{F})} " \operatorname{dom}\left(g_{0}^{\prime}\right) \subset^{*}$ $a_{\alpha} \cup g_{0}^{\prime}\left[a_{\alpha}\right]$ and $\operatorname{dom}\left(g_{1}^{\prime}\right) \subset^{*} c_{\alpha} \cup g_{1}^{\prime}\left[c_{\alpha}\right]$ ". For each $\gamma \in \lambda$, note that $\overrightarrow{f_{2}} \Vdash_{\mathbb{P}(\mathfrak{F})}$ " $a_{\gamma} \backslash a_{\alpha} \subset^{*} \mathbb{N} \backslash \operatorname{dom}\left(g_{0}^{\prime}\right)$ " and similarly $\vec{f}_{2} \Vdash_{\mathbb{P}(\mathfrak{F})} " c_{\gamma} \backslash c_{\alpha} \subset^{*} \mathbb{N} \backslash \operatorname{dom}\left(g_{1}^{\prime}\right)$ ".

Now consider the two disjoint sets: $Y_{0}=\bigcup_{k}\left[n_{6 k}, n_{6 k+3}\right)$ and its complement $Y_{1}$. Since $z$ is the $\mathbb{P}$-name of an ultrafilter, by possibly extending $\overrightarrow{f_{2}}=\left(f_{0}, f_{1}\right)$ even more, we may assume there is some $\beta>\alpha$ such that (by symmetry) $\vec{f}_{2} \Vdash_{\mathbb{P}}$ " $Y_{0} \subset^{*} Z_{\beta}$ ", in fact we may assume that $\vec{f}_{2} \Vdash_{\mathbb{P}} " F\left(Y_{0}\right) \subset^{*}$ $\left(L_{f_{0}} \times\{0\}\right) \cup\left(L_{f_{1}} \times\{1\}\right) "$.

Finally, let $\overrightarrow{f_{3}}=\left(f_{0}^{\prime}, f_{1}^{\prime}\right)<\vec{f}_{2}$ be chosen so that there is an infinite set $L \subset \mathbb{N}$ such that for $k \in L,\left[n_{6 k+1}, n_{6 k+2}\right) \subset \operatorname{dom}\left(f_{0}^{\prime}\right)$ and $\left[n_{6 k+1}, n_{6 k+2}\right) \not \subset$ $\operatorname{dom}\left(f_{0}\right)$. Set

$$
y=\bigcup_{k \in L}\left[n_{6 k+1}, n_{6 k+2}\right) \cap L_{f_{0}^{\prime}} \backslash L_{f_{0}}
$$

and choose any $\vec{f}_{4}<\vec{f}_{3}$ and $\widetilde{y}$ such that $\vec{f}_{4}$ forces that $F(\widetilde{y})=y \times\{0\}$. Since $\mathcal{J}$ is a dense ideal, we may fix any $J \in \mathcal{J}$ such that $J \cap \widetilde{y}$ is infinite.

It then follows that $\dot{h}[J \cap \widetilde{y}]={ }^{*} F(J \cap \widetilde{y}) \subset^{*} y \times\{0\}$, and so $J \cap \widetilde{y}$ is forced to be contained in $\bigcup_{k \in L}\left[n_{6 k}, n_{6 k+3}\right.$ ) (by the assumption on the sequence of $\left\{n_{k}\right\}$ 's). On the other hand, now that $J \cap \widetilde{y} \subset Y_{0}$, we have

$$
F(J \cap \widetilde{y}) \subset^{*} F\left(Y_{0}\right) \cap(\mathbb{N} \times\{0\}) \subset^{*} L_{f_{0}} \times\{0\},
$$

contradicting the fact that $y$ is disjoint from $L_{f_{0}}$. 


\section{Proof of Theorem 1.3}

TheOREM 5.1 (PFA). In the forcing extension by $\mathbb{P}_{1}$, a tie-point $x$ is introduced such that $\tau(x)=2$ and with $\mathbb{N}^{*}=A \bowtie B$, neither $A$ nor $B$ is a homeomorph of $\mathbb{N}^{*}$. In addition, there is no involution $F$ on $\mathbb{N}^{*}$ which has a unique fixed point, and so no tie-point is symmetric.

We begin by proving that neither $A$ nor $B$ can be homeomorphic to $\mathbb{N}^{*}$. We proceed much as in the previous section. Let $G \subset \mathbb{P}=\mathbb{P}_{1}$ be a generic filter. The tie-point $x$ as witnessed by $A, B$ will be the one given canonically by the $\mathbb{P}$-generic filter. More precisely, the closed set $A$ will be the closure of the union of the collection $\left\{\left(f^{-1}(0)\right)^{*}: f \in G\right\}$, while $B$ will be the closure of the union of the collection $\left\{f^{-1}(1): f \in G\right\}$. Of course, $x$ is the ultrafilter (a $P_{\omega_{2}}$-point) generated by the collection $\{\mathbb{N} \backslash \operatorname{dom}(f): f \in G\}$.

Assume there is an autohomeomorphism from $\mathbb{N}^{*}$ onto $A$ and let $F$ be any lifting, i.e. we may assume that there is an ultrafilter $z \in \mathbb{N}^{*}$ with dual ideal $\mathcal{I}$ such that $F$ is a function from $\bigcup_{f \in G}\left[f^{-1}(0)\right]^{\omega}$ onto $\bigcup_{I \in \mathcal{I}}[I]^{\omega}$ such that for each $f \in G, F\left\lceil\left[f^{-1}(0)\right]^{\omega}\right.$ is a lifting of a homeomorphism from $\left(f^{-1}(0)\right)^{*}$ onto $I_{f}^{*}$ for some $I_{f} \in \mathcal{I}$, and for each $I \in \mathcal{I}$, there is an $f \in G$ such that $I \subset^{*} I_{f}$.

Fix $\mathbb{P}$-names for all the above mentioned objects and apply Corollary 3.2 to find the filter $\mathcal{F} \subset \mathbb{P}$, the $\mathbb{P}$-name $\dot{h}$ and the sequence $\left\{n_{k}: k \in \omega\right\}$. We obtain a contradiction by showing there can be no such $\dot{h}$.

There is no loss of generality in this proof to assume that the $I$ mentioned in the statement of Corollary 3.2 is the empty set. The ideal denoted $\mathcal{J}$ as defined in the proof of Corollary 3.2 will now be generated by sets of the form $\bigcup\left\{\left[n_{k}, n_{k+1}\right) \cap f^{-1}(0):\left[n_{k}, n_{k+1}\right) \subset \operatorname{dom}(f)\right\}$ for $f \in \mathfrak{F}$. It follows that the ideal $\{F(J): J \in \mathcal{J}\}$ will be a dense ideal in $[\mathbb{N}]^{\omega}$. For each $J \in \mathcal{J}$, let $h_{J}$ denote the function on $J$ for which there is some $f_{J} \in \mathfrak{F}$ which forces that $h_{J}$ induces $F$ on $J$.

Since $\mathbb{P}(\mathfrak{F})$ is $\omega^{\omega}$-bounding, we may assume (by selecting a subsequence and renumbering) that the sequence $\left\{n_{k}: k \in \omega\right\}$ and some $f_{0} \in \mathfrak{F}$ satisfy:

(1) for each $i \in\left[n_{k}, n_{k+1}\right), f_{0} \Vdash_{\mathbb{P}(\mathfrak{F})}$ " $\dot{h}(i) \in\left[0, n_{k+2}\right)$ ",

(2) for each $i \in\left[n_{k}, n_{k+1}\right), f_{0} \Vdash_{\mathbb{P}(\mathfrak{F})}$ " $\dot{h}^{-1}(i) \in\left[0, n_{k+2}\right)$ ",

(3) for each $k$ there is an $m$ such that $n_{k}<2^{m}<2^{m+1}<n_{k+1}$, and $\left[2^{m}, 2^{m+1}\right) \backslash \operatorname{dom} f_{0}$ has at least $k$ elements.

We need a significant strengthening of Lemma 2.5 which holds for $\mathbb{P}=\mathbb{P}_{1}$.

Lemma 5.2. Assume that $h$ is a $\mathbb{P}(\mathfrak{F})$-name of a function from $\mathbb{N}$ into $\mathbb{N}$. Either there is an $f \in \mathfrak{F}$ such that $f \Vdash_{\mathbb{P}(\mathfrak{F})}$ " $h\lceil\operatorname{dom}(f) \notin V$ ", or there is an $f \in \mathfrak{F}$ and an increasing sequence $m_{1}<m_{2}<\cdots$ of integers such that $\mathbb{N} \backslash \operatorname{dom}(f)=\bigcup_{k} S_{k}$ where $S_{k} \subset 2^{m_{k+1}} \backslash 2^{m_{k}}$ and for each $i \in S_{k}$ the 
conditions $f \cup\{(i, 0)\}$ and $f \cup\{(i, 1)\}$ each force a value on $\dot{h}(i)$. Furthermore, if $f$ forces $h$ to be finite-to-one, we can arrange that for each $k$ and each $i \in$ $\left[n_{k}, n_{k+1}\right)$, either $f$ forces a value on $\dot{h}(i)$, or $f$ forces that $\dot{h}(i) \in\left[n_{k}, n_{k+1}\right)$.

Proof. First we choose $f_{0} \in \mathfrak{F}$ and some increasing sequence $n_{0}<n_{1}$ $<\cdots$ as in Lemma 2.5. We may choose, for each $k$, an $m_{k}$ such that $n_{k} \leq$ $2^{m_{k}}<2^{m_{k}+1}<n_{k+1}$ and $\lim _{k}\left|2^{m_{k}+1} \backslash\left(2^{m_{k}} \cup \operatorname{dom}\left(f_{0}\right)\right)\right|=\infty$. For each $k$, let $S_{k}^{0}=2^{m_{k}+1} \backslash\left(2^{m_{k}} \cup \operatorname{dom}\left(f_{0}\right)\right)$. By re-indexing we may assume that $\left|S_{k}^{0}\right| \geq k$, and we may arrange that $\mathbb{N} \backslash \operatorname{dom}\left(f_{0}\right)$ is equal to $\bigcup_{k} S_{k}^{0}$ and set $L_{0}=\mathbb{N}$. For each $k \in L_{0}$, let $i_{k}^{0}=\min S_{k}^{0}$ and choose any $f_{1}^{\prime}<f_{0}$ such that (by definition of $\mathbb{P}) I_{0}=\left\{i_{k}^{0}: k \in L_{0}\right\} \subset\left(f_{1}^{\prime}\right)^{-1}(0)$ and (by assumption on $\dot{h}$ ) $f_{1}^{\prime}$ forces a value on $\dot{h}\left(i_{k}^{0}\right)$ for each $k \in L_{0}$. Set $f_{1}=f_{1}^{\prime} \uparrow\left(\mathbb{N} \backslash I_{0}\right)$ and for each $k \in L_{0}$, let $S_{k}^{1}=S_{k}^{0} \backslash\left(\left\{i_{k}^{0}\right\} \cup \operatorname{dom}\left(f_{1}\right)\right)$. By further extending $f_{1}$ we may also assume that $f_{1} \cup\left\{\left(i_{k}^{0}, 1\right)\right\}$ also forces a value on $\dot{h}\left(i_{k}^{0}\right)$. Choose $L_{1} \subset L_{0}$ such that $\lim _{k \in L_{1}}\left|S_{k}^{1}\right|=\infty$. Notice that each $i_{k}^{0}$ is the minimum element of $S_{k}^{1}$. Again, we may extend $f_{1}$ and assume that $\mathbb{N} \backslash \operatorname{dom}\left(f_{1}\right)$ is equal to $\bigcup_{k \in L_{1}} S_{k}^{1}$. Suppose now we have some infinite $L_{j}$, some $f_{j}$, and for $k \in L_{j}$, an increasing sequence $\left\{i_{k}^{0}, i_{k}^{1}, \ldots, i_{k}^{j-1}\right\} \subset S_{k}^{0}$. Assume further that

$$
S_{k}^{j} \cup\left\{i_{k}^{l}: l<j\right\}=S_{k}^{0} \backslash \operatorname{dom}\left(f_{j}\right)
$$

and that $\lim _{k \in L_{j}}\left|S_{k}^{j} \backslash i_{k}^{j-1}\right|=\infty$. For each $k \in L_{j}$, let $i_{k}^{j}=\min \left(S_{k}^{j} \backslash\left\{i_{k}^{l}\right.\right.$ : $l<j\}$ ). By a simple recursion of length $2^{j}$, there is an $f_{j+1}<f_{j}$ such that, for each $k \in L_{j},\left\{i_{k}^{l}: l \leq j\right\} \subset S_{k}^{0} \backslash \operatorname{dom}\left(f_{j+1}\right)$ and for each function $s$ from $\left\{i_{k}^{l}: l \leq j\right\}$ into 2 , the condition $f_{j+1} \cup s$ forces a value on $\dot{h}\left(i_{k}^{j}\right)$. Again find $L_{j+1} \subset L_{j}$ so that $\lim _{k \in L_{j+1}}\left|S_{k}^{j+1}\right|=\infty\left(\right.$ where $\left.S_{k}^{j+1}=S_{k}^{0} \backslash \operatorname{dom}\left(f_{j+1}\right)\right)$ and extend $f_{j+1}$ so that $\mathbb{N} \backslash \operatorname{dom}\left(f_{j+1}\right)$ is equal to $\bigcup_{k \in L_{j+1}} S_{k}^{j+1}$.

We are half-way there. At the end of this fusion, the function $\bar{f}=\bigcup_{j} f_{j}$ is a member of $\mathbb{P}$ because for each $j$ and $k \in L_{j+1}, 2^{m_{k}+1} \backslash\left(2^{m_{k}} \cup \operatorname{dom}(\bar{f})\right) \supset$ $\left\{i_{k}^{0}, \ldots, i_{k}^{j}\right\}$. For each $k$, let $\bar{S}_{k}=S_{k}^{0} \backslash \operatorname{dom}(\bar{f})$; by possibly extending $\bar{f}$, we may again assume that there is some $L$ such that $\lim _{k \in L}\left|\bar{S}_{k}\right|=\infty$. What we have proven about $\bar{f}$ is that for each $k \in L$ and each $i \in \bar{S}_{k}$ and each function $s$ from $i \cap \bar{S}_{k}$ to $2, \bar{f} \cup s \cup\{(i, 0)\}$ and $\bar{f} \cup\{(i, 1)\}$ each force a value on $\dot{h}(i)$. By the genericity of $\mathfrak{F}$, there must be such a condition as $\bar{f}$ in $\mathfrak{F}$.

To finish, simply repeat the same process as above except this time choose maximal values and work down the values in $\bar{S}_{k}$. That is, there will be an infinite set $K$ and a condition $f^{\dagger}$ such that for each $k \in K$, there is a decreasing sequence $\left\{i_{k}^{0}, i_{k}^{1}, \ldots, i_{k}^{j_{k}}\right\} \subset \bar{S}_{k} \backslash \operatorname{dom}\left(f^{\dagger}\right)$ with $\lim _{k}\left\{j_{k}: k \in K\right\}$ $=\infty$. These will have the property that for each $k \in K$ and $j \leq j_{k}$ and each function $s:\left\{i_{k}^{0}, \ldots, i_{k}^{j-1}\right\} \rightarrow 2$, each of $f^{\dagger} \cup s \cup\left\{\left(i_{k}^{j}, 0\right)\right\}$ and $f^{\dagger} \cup s \cup\left\{\left(i_{k}^{j}, 1\right)\right\}$ will force a value on $\dot{h}\left(i_{k}^{j}\right)$. 
Now we show that $f^{\dagger} \cup\left\{\left(i_{k}^{j}, e\right)\right\}(e \in 2)$ forces a value on $\dot{h}\left(i_{k}^{j}\right)$ as required. If it did not, then we could find extensions $f_{0}, f_{1}$ of $f^{\dagger} \cup\left\{\left(i_{k}^{j}, e\right)\right\}$ which force different values on $\dot{h}\left(i_{k}^{j}\right)$. Let $s_{0}=f_{0}\left\lceil S_{0}^{k} \cap i_{k}^{j}\right.$ and $s_{1}=f_{1}\left\lceil S_{0}^{k} \backslash i_{k}^{j}\right.$. Notice that $\bar{f} \cup s_{0} \leq f^{\dagger} \cup s_{0}$ forces a value (hence the same value as that forced by $f_{0}$ ) on $\dot{h}\left(i_{k}^{j}\right)$. This is also true for $f^{\dagger} \cup s_{1}$ in that it forces the same value on $\dot{h}\left(i_{k}^{j}\right)$ as that forced by $f_{1}$. The contradiction is that $\bar{f} \cup s_{0}$ and $f^{\dagger} \cup s_{1}$ force distinct values on $\dot{h}\left(i_{k}^{j}\right)$ although they have the common extension $f^{\dagger} \cup s_{0} \cup s_{1}$.

Returning to the proof of Theorem 5.1, we are ready to use Lemma 5.2 to show that forcing with $\mathbb{P}(\mathfrak{F})$ will not introduce undesirable functions $h$, analogously to the argument in Theorem 1.1. By Lemma 5.2, we have the condition $f_{0} \in \mathfrak{F}$ and the sequence $S_{k}(k \in \mathbb{N})$ such that $\mathbb{N} \backslash \operatorname{dom}\left(f_{0}\right)=\bigcup_{k} S_{k}$ and that for each $i \in \bigcup_{k} S_{k}, f_{0} \cup\{(i, 0)\}$ forces a value (call it $\bar{h}(i)$ ) on $\dot{h}(i)$. Therefore, $\bar{h}$ is a function with domain $\bigcup_{k} S_{k}$ in $V$. We may assume that $\left|S_{k}\right| \geq k$ for each $k$. It suffices to find a condition in $\mathbb{P}$ below $f_{0}$ which forces that there is some $J \in \mathcal{J}$ such that $h_{J}$ is not extended by $\dot{h}$. It is useful to note that if $Y \subset \bigcup_{k} S_{k}$ is such that $\lim \sup \left|S_{k} \backslash Y\right|$ is infinite, then for any function $g \in 2^{Y}, f_{0} \cup g \in \mathbb{P}$.

We first check that $\bar{h}$ is 1-to-1 on a cofinite set. If not, there is an infinite set of pairs $E_{j} \subset \bigcup_{k} S_{k}, \bar{h}\left[E_{j}\right]$ is a singleton and such that for each $k$, $S_{k} \cap \bigcup_{j} E_{j}$ has at most two elements. Let $L$ denote the set of $k$ for which $S_{k}$ meets $\bigcup_{j} E_{j}$. By passing to a subcollection of the $E_{j}$ 's we may assume that $L$ has infinite complement. Let $g$ be the function with domain $\bigcup_{j} E_{j}$ which is constantly 0 . Then $f_{0} \cup g$ forces that $\dot{h}$ agrees with $\bar{h}$ on $\operatorname{dom}(g)$ and so is not 1-to-1. There is a further extension $f_{1}$ of $f_{0} \cup g$ with the property that $S_{k} \subset \operatorname{dom}\left(f_{1}\right)$ for all $k \notin L$. Therefore, by virtue of $f_{1}$, there is some $J \in \mathcal{J}$ which contains $\bigcup_{j} E_{j}$. However, this is a contradiction, because apparently $h_{J}=\dot{h}\left\lceil J\right.$ does not induce a homeomorphism on $J^{*}$.

But now that we know that $\bar{h}$ is 1-to-1 we can get a contradiction as follows. Let $f_{1}<f_{0}$ be chosen so as to decide the value of $F\left(f_{0}^{-1}(0)\right)$, and let $Y$ denote this value. For each $k$, let $\bar{S}_{k}=S_{k} \backslash \operatorname{dom}\left(f_{1}\right)$ and let $L$ be such that $\left\{\left|\bar{S}_{k}\right|: k \in L\right\}$ diverges to infinity. If $Y \cap \bar{h}\left[\bigcup_{k \in L} \bar{S}_{k}\right]$ is infinite, then there is an infinite set $L_{0} \subset L$ (with $L \backslash L_{0}$ also infinite) such that for each $k \in L_{0}$, there is an $i_{k} \in \bar{S}_{k}$ such that $\bar{h}\left(i_{k}\right) \in Y$. Choose any $f_{2}<f_{1}$ such that $f_{2}\left(i_{k}\right)=0$ and $S_{k} \subset \operatorname{dom}\left(f_{2}\right)$ for all $k \in L_{0}$. It follows that there is a $J \in \mathcal{J}$ with $\bigcup_{k \in L_{0}} S_{k} \cap f_{2}^{-1}(0) \subset J$ and such that

$$
f_{2} \Vdash_{\mathbb{P}} \text { " } F\left(\left\{i_{k}: k \in L_{0}\right\}\right) \cap F\left(f_{0}^{-1}(0)\right)={ }^{*} h_{J}\left[\left\{i_{k}: k \in L_{0}\right\}\right] \cap Y \text { is infinite", }
$$

a contradiction since $\left\{i_{k}: k \in L_{0}\right\}$ is disjoint from $f^{-1}(0)$. On the other hand, let $L$ be as above and $L_{0}$ any infinite-coinfinite subset. Fix any sequence $\left\{i_{k}: k \in L_{0}\right\}$ (with each $i_{k} \in \bar{S}_{k}$ ) and select $f_{2}<f_{1}$ so that $f_{2}\left(i_{k}\right)=1$ for all $k \in L_{0}$ and $\bigcup_{k \in L_{0}} S_{k} \subset \operatorname{dom}\left(f_{2}\right)$. Set $Y^{\prime}=\bar{h}\left[\left\{i_{k}: k \in L_{0}\right\}\right]$. Since $\bar{h}$ 
is 1-to-1 it follows easily that $f_{2} \Vdash_{\mathbb{P}}$ " $(\forall J \in \mathcal{J}) F(J) \cap Y^{\prime}$ is finite". This, of course, is also a contradiction.

Now we consider the possibility that $\tau(x)>2$. It then follows that one of $A \backslash\{x\}$ or $B \backslash\{x\}$, say the former, can be partitioned into disjoint clopen non-compact sets. Therefore there is some sequence $\left\{c_{\alpha}: \alpha \in \omega_{2}\right\}$ of $\mathbb{P}$-names such that for each $\alpha<\beta \in \omega_{2}, c_{\beta} \subset a_{\beta}$ and $c_{\beta} \cap a_{\alpha}={ }^{*} c_{\alpha}$. In addition, for each $\alpha<\omega_{2}$ there must be a $\beta \in \omega_{2}$ such that $c_{\beta} \backslash a_{\alpha}$ and $a_{\beta} \backslash\left(c_{\beta} \cup a_{\alpha}\right)$ are both infinite.

In this case, we suppose that $H$ and $\mathfrak{F}$ are chosen as in Lemma 2.2, and in the extension by $H$, let $\lambda$ denote the ordinal $\omega_{2}$ from $V$. In this model we will have a $(\lambda, \lambda)$-gap formed by the families $\left\{c_{\alpha}: \alpha \in \lambda\right\}$ and $\left\{a_{\alpha} \backslash c_{\alpha}: \alpha \in \lambda\right\}$. Assume that we can show that in the extension obtained by forcing with $\mathbb{P}(\mathfrak{F})$, there is no $C \subset \mathbb{N}$ such that $C \cap a_{\alpha}={ }^{*} c_{\alpha}$ for all $\alpha \in \lambda$. In other words, for any cofinal sequence $\left\{\alpha_{\xi}: \xi \in \omega_{1}\right\} \subset \lambda$, the collections $\left\{c_{\alpha_{\xi}}, a_{\alpha_{\xi}} \backslash c_{\alpha_{\xi}}: \xi \in \omega_{1}\right\}$ form an $\left(\omega_{1}, \omega_{1}\right)$-gap. There are wellknown ccc posets $Q_{1}$ (see $[1,4.2]$ ) which "freeze" the gap. What we mean here is that there is a family of $\omega_{1}$-many dense subsets of the iteration $\omega_{2}^{<\omega_{1}} * \mathbb{P}(\mathcal{F}) * Q_{1}$ such that if a filter meets them all, then the gap will remain a gap in any proper forcing extension. Finally, if we let $Q_{2}$ be the $\sigma$-centered poset mentioned after Definition 1.6, there is a filter (meeting $\omega_{1}$-many dense subsets) on the proper iteration $\omega_{2}^{<\omega_{1}} * \mathbb{P}(\mathcal{F}) * Q_{1} * Q_{2}$ which introduces a condition $f \in \mathbb{P}$ which forces that $c_{\lambda}$ will not exist.

Thus, we will have shown that $\tau(x)=2$ once we show that there is no $\mathbb{P}(\mathfrak{F})$-name for a set $C$ as above. Equivalently, we assume that $\dot{h}$ is a $\mathbb{P}(\mathfrak{F})$ name for the characteristic function of $\mathbb{N} \backslash C$, and derive a contradiction.

So, given our name $\dot{h}$, we repeat the steps above up to the point where we have $f_{0}$ and the sequence $\left\{S_{k}: k \in \mathbb{N}\right\}$ so that $f_{0} \cup\{(i, 0)\}$ forces a value $\bar{h}(i)$ on $\dot{h}(i)$ for each $i \in \bigcup_{k} S_{k}$ and $\mathbb{N} \backslash \operatorname{dom}\left(f_{0}\right)=\bigcup_{k} S_{k}$.

Let $Y=\bar{h}^{-1}(0)$ and $Z=\bar{h}^{-1}(1)$. Since $x$ is forced to be an ultrafilter, there is an $f_{1}<f_{0}$ such that $\operatorname{dom}\left(f_{1}\right)$ contains one of $Y$ or $Z$. If $\operatorname{dom}\left(f_{1}\right)$ contains $Y$, then $f_{1}$ forces that $\dot{h}\left[a_{\beta} \backslash \operatorname{dom}\left(f_{1}\right)\right]=1$, and so $a_{\beta} \backslash \operatorname{dom}\left(f_{1}\right) \subset^{*}$ $\mathbb{N} \backslash C$ for all $\beta \in \omega_{2}$. While if $\operatorname{dom}\left(f_{1}\right)$ contains $Z$, then $f_{1}$ forces that $\dot{h}\left[a_{\beta} \backslash \operatorname{dom}\left(f_{1}\right)\right]=0$, and so $a_{\beta} \backslash \operatorname{dom}\left(f_{1}\right) \subset^{*} C$ for all $\beta \in \omega_{2}$. However, taking $\beta$ so large that each of $c_{\beta} \backslash \operatorname{dom}\left(f_{1}\right)$ and $a_{\beta} \backslash\left(c_{\beta} \cup \operatorname{dom}\left(f_{1}\right)\right)$ are infinite shows that no such $\dot{h}$ exists.

Finally, we show that there are no involutions on $\mathbb{N}^{*}$ which have a unique fixed point. Assume that $\Phi$ is such an involution and that $y$ is the unique fixed point of $\Phi$. Let $F$ be an arbitrary lifting of $\Phi$ to $[\mathbb{N}]^{\omega}$. Let $\mathcal{I}$ denote the dual ideal to $y$ ). We first show that $\mathcal{I}$ is a $P$-ideal (i.e. that $y$ is a $P$-point). For each $I \in \mathcal{I}, F(I)$ is also in $\mathcal{I}$ and $F(I \cup F(I))={ }^{*} I \cup F(I)$. So we may let $\mathcal{Z}$ denote those $I \in \mathcal{I}$ such that $Z={ }^{*} F(Z)$. Given $Z \in \mathcal{Z}$, since 
fix $(\Phi) \cap Z^{*}=\emptyset$, there is a collection $\mathcal{Y} \subset[Z]^{\omega}$ such that $F(Y) \cap Y={ }^{*} \emptyset$ for each $Y \in \mathcal{Y}$, and such that $Z^{*}$ is covered by $\left\{Y^{*}: Y \in \mathcal{Y}\right\}$. By compactness, we may assume that $\mathcal{Y}=\left\{Y_{0}, \ldots, Y_{n}\right\}$ is finite. Set $Z_{0}=Y_{0} \cup F\left(Y_{0}\right)$. By induction, replace $Y_{k}$ by $Y_{k} \backslash \bigcup_{j<k} Z_{j}$ and define $Z_{k}=Y_{k} \cup F\left(Y_{k}\right)$. Therefore $Y_{Z}=\bigcup_{k} Y_{k}$ satisfies $Y_{Z} \cap F\left(Y_{Z}\right)={ }^{*} \emptyset$ and $Z=Y_{Z} \cup F\left(Y_{Z}\right)$. This shows that for each $Z \in \mathcal{Z}$ there is a partition of $Z=Z^{0} \cup Z^{1}$ such that $F\left(Z^{0}\right)={ }^{*} Z^{1}$. We can show $y$ is a $P$-point. Indeed, if $\left\{Z_{n}=Z_{n}^{0} \cup Z_{n}^{1}: n \in \mathbb{N}\right\} \subset \mathcal{Z}$ are pairwise disjoint, then $y \notin \overline{\bigcup_{n} Z_{n}^{*}}$ since $F\left(\overline{\bigcup_{n}\left(Z_{n}^{0}\right)^{*}}\right)=\overline{\bigcup_{n}\left(Z_{n}^{1}\right)^{*}}$ and $\overline{\bigcup_{n}\left(Z_{n}^{0}\right)^{*}}$ is disjoint from $\overline{\bigcup_{n}\left(Z_{n}^{1}\right)^{*}}$.

Fix $\mathbb{P}$-names for $F$ and the members of $\mathcal{I}$ and let $H, \mathcal{F}, F$ and $\left\{n_{k}\right.$ : $k \in \omega\}$ be as given in Lemma 3.1. Also let $\mathcal{J}$ denote the ideal as defined in the proof of Corollary 3.2. Hence $J \in \mathcal{J}$ if there is an $f \in \mathcal{F}$ and $J \subset$ $\bigcup\left\{\left[n_{k}, n_{k+1}\right):\left[n_{k}, n_{k+1}\right) \subset \operatorname{dom}(f)\right\}$. It is again easily argued that the $I \in \mathcal{I}$ as specified in Corollary 3.2 can be assumed to be empty. For each $J \in \mathcal{J}$, let $h_{J}$ be the function on $J$ such that there is an $f \in \mathcal{F}$ which forces that $h_{J}$ induces $F$ on $J$. Let $\dot{h}$ be the $\mathbb{P}(\mathcal{F})$-name as given in Corollary 3.2. Since $F$ is an involution with a unique fixed point, we may assume that $\dot{h}$ is forced to satisfy that $\dot{h}(\dot{h}(i))=i \neq \dot{h}(i)$ for all $i$.

The rest of the proof depends on the following modification of Lemma 5.2 .

Claim 4. There is an $f \in \mathfrak{F}$ and a sequence of sets $\left\{m_{k}, S_{k}, T_{k}: k \in \omega\right\}$ and mappings $\psi_{k}: T_{k} \rightarrow S_{k}$ such that $S_{k} \subset 2^{m_{k}+1} \backslash 2^{m_{k}} \subset\left[n_{k}, n_{k+1}\right)$, $T_{k} \subset\left[n_{k}, n_{k+1}\right), \mathbb{N} \backslash \operatorname{dom}(f)=\bigcup_{k} S_{k}$, and for each $k$ and $i \in S_{k}$ and $\bar{f}<f$, $\bar{f}$ forces a value on $\bar{h}\left\lceil\psi_{k}^{-1}(i)\right.$ iff $i \in \operatorname{dom}(\bar{f})$.

Before proving the claim, let us show how this will finish the proof. For each $i \notin \operatorname{dom}(f)$, there are two functions $h_{i}^{0}, h_{i}^{1}$ with domain $\psi^{-1}(i)$ such that $f \cup\{\langle i, e\rangle\}$ forces that $h_{i}^{e} \subset \dot{h}$. Since, by assumption, for each $i \in \operatorname{dom}(f), f$ does not already force a value on $\dot{h}\left\lceil\psi^{-1}(i)\right.$, we can choose $j_{i} \in \psi^{-1}(i)$ such that $v_{i}=h_{i}^{0}\left(j_{i}\right) \neq h_{i}^{1}\left(j_{i}\right)=w_{i}$. Note that, by our assumption on $\dot{h}$, it also follows that $\psi\left(v_{i}\right)=\psi\left(w_{i}\right)=i$.

Choose $g<f$ which forces a value, $Y$, on $F\left(\left\{j_{i}\right\}_{i \notin \operatorname{dom}(f)}\right)$. Assume that $Y \cap\left\{v_{i}: i \notin \operatorname{dom}(g)\right\}$ is infinite. It follows easily that there is some $g^{\dagger}<g$ such that $Y \cap\left\{v_{i}: g^{\dagger}(i)=1\right\}$ is infinite and let $J \subset\left\{i \in \operatorname{dom}\left(g^{\dagger}\right): g^{\dagger}(i)=1\right.$ and $\left.v_{i} \in Y\right\}$ be any infinite set such that $\left\{j_{i}\right\}_{i \in J}$ is in $\mathcal{J}$. However, this is a contradiction since

$$
g^{\dagger} \Vdash_{\mathbb{P}} " F "\left(\left\{j_{i}: i \in J\right\}\right)={ }^{*} \dot{h}\left[\left\{j_{i}\right\}_{i \in J}\right]=\left\{w_{i}\right\}_{i \in I} \subset^{*} Y \cap\left\{v_{i}\right\}_{i \in J} .
$$

The argument when $\left\{v_{i}: i \notin \operatorname{dom}(g)\right\} \backslash Y$ is infinite is similar.

Now we prove Claim 4. For any $k$, condition $g$, and $T \subset\left[n_{k}, n_{k+1}\right)$ let $\operatorname{Orb}(T, g)$ denote the set $\left\{j:\left(\exists g^{\prime}<g\right)(\exists t \in T) g^{\prime} \Vdash_{\mathbb{P}(\mathfrak{F})}\right.$ " $\left.\dot{h}(t)=j "\right\}$. Fix 
any $k$ and the $f$ as above that was selected from Lemma 2.5. Let $g_{0}=$ $f\left\lceil\left[n_{k}, n_{k+1}\right)\right.$ and assume, as we may, that $S_{0}^{k}=\left[n_{k}, n_{k+1}\right) \backslash \operatorname{dom}\left(g_{0}\right)$ is contained in $\left[2^{m}, 2^{m+1}\right)$ for some $m$. By a simple recursion much as in Lemma 5.2 , we can choose increasing sequences $I_{l}=\left\{i_{0}, i_{1}, \ldots, i_{l-1}\right\} \subset S_{0}^{k}$ and extensions $g_{l} \supset g_{l-1} \supset \cdots \supset g_{0}$ so that $I_{l} \subset S_{l}^{k}=S_{0}^{k} \backslash \operatorname{dom}\left(g_{l}\right)$ and $i_{l}=$ $\min \left(S_{l}^{k} \backslash I_{l}\right)$. In addition, select sets $T\left(i_{l}\right) \subset\left[n_{k}, n_{k+1}\right) \backslash \bigcup_{j<l} \operatorname{Orb}\left(T\left(i_{j}\right), g_{l}\right)$ of minimum cardinality (at most $2^{l}$ ) so that for each $s: S_{l}^{k} \rightarrow 2$ there is, if possible, a $t \in T\left(i_{l}\right)$ such that $s \cup g_{l} \cup\left\{\left(i_{j}, 0\right)\right\}$ and $s \cup g_{l} \cup\left\{\left(i_{j}, 1\right)\right\}$ force distinct values on $\dot{h}(t)$. Notice that $\operatorname{Orb}\left(\{t\}, g_{l}\right)$ has cardinality at most $2^{l}$ for each $t \in \bigcup_{j<l} T\left(i_{j}\right)$. We also require that for each $s: S_{l}^{k} \rightarrow 2$, each of $s \cup\left\{\left(i_{l}, 0\right)\right\} \cup g_{l+1}$ and $s \cup\left\{\left(i_{l}, 1\right)\right\} \cup g_{l+1}$ force a value on $\dot{h}_{\uparrow} \operatorname{Orb}\left(T\left(i_{l}\right), g_{l+1}\right)$. If $T\left(i_{l}\right)$ is not empty, we can certainly ensure that for at least one $s: S_{l}^{k} \rightarrow 2$ and $t \in T\left(i_{l}\right), s \cup\left\{\left(i_{l}, 1\right)\right\} \cup g_{l+1}$ forces a distinct value on $\dot{h}(t)$ from that forced by $s \cup\left\{\left(i_{l}, 0\right)\right\} \cup g_{l+1}$.

For each successive $l$, there is a recursion on $k$ so that $f_{l}=f \cup \bigcup\left\{g_{l}^{k}\right.$ : $k \in \omega\}$ is a condition. If for each $k$, there is an $s^{k}: I_{l}^{k} \rightarrow 2$ for which no suitable $t \in\left[n_{k}, n_{k+1}\right)$ can be chosen, then it is because the condition $g=$ $f_{k} \cup \bigcup_{k}\left(s_{k} \cup g_{j}^{k}\right)$ forces a value on $\dot{h}(t)$ for all $t \notin \bigcup_{k} \bigcup_{j<l} \operatorname{Orb}\left(T\left(i_{k}^{j}\right), g\right)$. But if this were the case, then this condition would force a value on $\dot{h}(t)$ for all $t$.

After infinitely many steps, we may instead assume that (a new choice of) $f$ simply has this property: for each $k$ and each $S^{k}=\left[2^{m_{k}}, 2^{m_{k}+1}\right) \backslash \operatorname{dom}(f)=$ $\left[n_{k}, n_{k+1}\right) \backslash \operatorname{dom}(f)$, there is a sequence $\left\{T(i): i \in S^{k}\right\}$ of pairwise disjoint finite subsets of $\left[n_{k}, n_{k+1}\right)$ such that for each $i \in S^{k}$ and each $s: S^{k} \cap i \rightarrow 2$, $s \cup\{(i, 0)\} \cup f$ and $s \cup\{(i, 1)\} \cup f$ each force a value on $\dot{h}\lceil T(i)$ while $s \cup f$ does not. (We do not need a superscript on the $T$ 's since they depend only on $i$ and not on $k$.) We have also ensured that for $i \neq i^{\prime}, \operatorname{Orb}(T(i), f)$ is disjoint from $\operatorname{Orb}\left(T\left(i^{\prime}\right), f\right)$.

Now, much as in Lemma 5.2, we repeat the process but rather than choosing minimal members of $S^{k}$ we choose maximal. A new trouble arises in this proof because of the sizes of the sets $T(i)$, while in Lemma 5.2, each $T(i)$ was just $\{i\}$. To overcome this, we will use the next claim.

Claim 5. For each $f_{1}<f$ and infinite $I \subset \mathbb{N} \backslash \operatorname{dom}\left(f_{1}\right)$ and $K \subset \mathbb{N}$ for which $\left\{\left|I \cap S^{k}\right|: k \in K\right\}$ diverges to infinity, there is an $f_{2}<f_{1}$, $I^{\prime} \subset I \backslash \operatorname{dom}\left(f_{2}\right)$, and $K^{\prime} \subset K$ such that $\left\{\left|I^{\prime} \cap S^{k} \backslash \operatorname{dom}\left(f_{2}\right)\right|: k \in K^{\prime}\right\}$ diverges to infinity, and for all $i \in I^{\prime}$, each of $f_{2} \cup\{(i, 0)\}$ and $f_{2} \cup\{(i, 1)\}$ force a value on $\dot{h} \uparrow T(i)$.

In order to not lose track of our progress, let us again defer the proof of Claim 5 and first finish the proof of Claim 4 .

Let $K_{0} \subset \omega$ be chosen so that $\left\{\left|S^{k}\right|: k \in K_{0}\right\}$ is strictly increasing. By Claim 5 there is an infinite $K_{1} \subset K_{0}$ and an $f_{1}<f$ so that for each $k \in K_{1}$, 
there is an $i_{0}^{k} \notin \operatorname{dom}\left(f_{1}\right)$ such that $f_{1} \cup\left\{\left(i_{0}^{k}, 0\right)\right\}$ and $f_{1} \cup\left\{\left(i_{0}^{k}, 1\right)\right\}$ each force a value on $\dot{h}\left\lceil T\left(i_{0}^{k}\right)\right.$, and $\left|S^{k} \cap i_{0}^{k}\right|>\left|S^{k}\right| / 2$.

By induction on $j>0$, continue to choose $f_{j}<f_{j-1}, i_{j}^{k} \in S^{k} \cap i_{j-1}^{k} \backslash$ $\operatorname{dom}\left(f_{j}\right)$ for all $k$ in an infinite set $K_{j} \subset K_{j-1}$ such that the sequence $\left\{\left|\left(S^{k} \cap i_{j}^{k}\right) \backslash \operatorname{dom}\left(f_{j-1}\right)\right|: k \in K_{j}\right\}$ diverges to infinity. We require that for each $k \in K_{j}$ and $s:\left\{i_{l}^{k}: l \leq j\right\} \rightarrow 2$, the condition $s \cup f_{j}$ forces a value on $\dot{h} \uparrow T\left(i_{j}^{k}\right)$.

We find the sequence $\left\{i_{j}^{k}: k \in K_{j}\right\}$ by applying Claim 5 as follows. For each function $\psi: j \rightarrow 2$ and each $k \in K_{j-1}$, let $s_{\psi}^{k}$ denote the function from $\left\{i_{0}^{k}, \ldots, i_{j-1}^{k}\right\}$ to 2 such that $s_{\psi}^{k}\left(i_{j^{\prime}}^{k}\right)=\psi\left(j^{\prime}\right)$ for each $j^{\prime}<j$. Start by applying Claim 5 with the $f_{1}$ in Claim 5 being $f_{j-1} \cup \bigcup_{k \in K_{j-1}} s_{\psi}^{k}$ for some fixed $\psi \in 2^{j}, K=K_{j-1}$ and, $I=\bigcup_{k \in K_{j-1}} S^{k} \backslash \operatorname{dom}\left(f_{1}\right)$. Simply apply Claim 5 recursively, each time swapping the values of the $f_{1}$ used so as to cycle through all the possible $\psi \in 2^{j}$. After these $2^{j}$ steps, each time shrinking the $K^{\prime}$ and the $I^{\prime}$ we can let $f_{j}$ be the final condition denoted $f_{2}$ in Claim $5, K_{j}$ be the final set $K^{\prime}$ and let $\left\{i_{j}^{k}: k \in K_{j}\right\}$ be any selection from the final $I^{\prime}$ which has the additional property that $\left\{\left|S^{k} \cap i_{j}^{k}\right|: k \in K_{j}\right\}$ diverges to infinity.

What we have now is that for each $k \in K_{j}$ and each $\psi \in 2^{j}$, the conditions $s_{\psi}^{k} \cup f_{j} \cup\left\{\left(i_{j}^{k}, 0\right)\right\}$ and $s_{\psi}^{k} \cup f_{j} \cup\left\{\left(i_{j}^{k}, 1\right)\right\}$ each force a value on $\dot{h}\left\lceil T\left(i_{j}^{k}\right)\right.$.

When this recursion finishes, let $\left\{k_{j}: j \in \omega\right\}$ be chosen so that $k_{j} \in K_{j}$ for each $j$. Set $\bar{f} \supset \bigcup_{j} f_{j} \uparrow\left[n_{k_{j}}, n_{k_{j}+1}\right)$. Note that for each $j, k=k_{j}$ and $l<j$, $i_{l}^{k} \notin \operatorname{dom}(\bar{f})$ and $\bar{f}$ is not forcing a value on $\dot{h} \uparrow T\left(i_{l}^{k}\right)$. Assume $g<\bar{f}, k=k_{j}$ and $l<j$. Let $s=g \uparrow\left(S^{k} \cap i_{l}^{k}\right)$ and $s^{\prime}=g \uparrow\left(S^{k} \backslash 1+i_{l}^{k}\right)$.

There is a $t \in T\left(i_{l}^{k}\right)$ such that $s \cup f_{j} \cup\left\{\left(i_{l}^{k}, 0\right)\right\}$ and $s \cup f_{j} \cup\left\{\left(i_{l}^{k}, 1\right)\right\}$ force different values on $\dot{h}(t)$. Therefore if $i_{l}^{k} \notin \operatorname{dom}(g)$, then $g$ cannot decide $\dot{h}(t)$. On the other hand, suppose $i_{l}^{k}$ is in $\operatorname{dom}(g)$, let $e=g\left(i_{l}^{k}\right)$ and assume that there is a $t \in T\left(i_{j}^{k}\right)$ such that $\dot{h}(t)$ is not decided by $g$. Fix any $s_{1}:\left\{i_{j}^{k}: j<l\right\}$ $\rightarrow 2$ extending $s^{\prime}$ such that $s_{1} \cup g$ forces a value on $\dot{h}(t)$ and let $v$ be this value. Since $g$ does not decide $\dot{h}(t)=v$, there is some $s_{2}: S^{k} \cap i_{j}^{k} \rightarrow 2$ which extends $s$ and forces a value distinct from $v$ on $\dot{h}(t)$. This is a contradiction since $s_{1} \cup s_{2} \cup g$ is a condition.

We have shown that for each $k_{j}$ and $l<j, g$ forces a value on $\dot{h}\left\lceil T\left(i_{l}^{k}\right)\right.$ iff $i_{l}^{k} \in \operatorname{dom}(g)$.

It remains to give the following proof.

Proof of Claim 5. Let $f_{1}$ and $I$ be as in the statement of the claim and assume there is no such $f_{2}$ and $I^{\prime}$. Let $\mathfrak{I}=\left\{I^{\prime} \in[I]^{\omega}:\left\{\left|I^{\prime} \cap S^{k}\right|\right\}_{k}\right.$ is bounded $\}$ and $\mathfrak{I}^{*}=\left\{I^{\prime} \in[I]^{\omega}:\left\{\left|I^{\prime} \cap S^{k}\right|\right\}_{k}\right.$ diverges to infinity $\}$. For each $I^{\prime} \subset I$, let $K\left(I^{\prime}\right)=\left\{k: I^{\prime} \cap S^{k} \neq \emptyset\right\}$. For any set $I$, let $\chi_{0}(I)$ (respectively $\chi_{1}(I)$ ) denote the function which is constantly 0 (respectively 1 ) on $I$. 
Choose, if possible, $e \in\{0,1\}$ (say $e=0$ ) and some pair $f_{2}<f_{1}$ and $I_{2} \subset I \backslash \operatorname{dom}\left(f_{2}\right)$ such that $I_{2} \in \mathfrak{I}^{*}$ and $f_{2} \cup \chi_{0}\left(I_{2}\right)$ forces a value on $\dot{h} \uparrow T(i)$ for all $i \in I_{2}$. If no such $e$ exists, then let $f_{2}=f_{1}$ and $I_{2}=I$. It now follows (in either case) that for any $f_{3}<f_{2}$ and $I^{\prime} \subset I_{2}$, the set of $i \in I^{\prime}$ for which $f_{3} \uparrow\left(\operatorname{dom}\left(f_{3}\right) \backslash I^{\prime}\right) \cup \chi_{1}\left(I^{\prime}\right)$ forces a value on $\dot{h}(i)$ is a member of $\mathfrak{I}$.

For each integer $k$, let $\mathcal{S}_{k}$ be the set of partial functions from $S^{k}$ into 2 . For integers $l, k$ and condition $g$, let

$$
\mathcal{S}(l, k, g)=\left\{s \in \mathcal{S}_{k}: g \mid S^{k} \subset s \text { and }\left|S^{k} \backslash \operatorname{dom}(s)\right|>l\right\} .
$$

For $s \in \mathcal{S}\left(l, k, f_{2} \cup \chi_{1}\left(I_{2}\right)\right)$, let $I(s)$ be the set of $i \in I_{2} \cap S^{k}$ such that $s \cup f_{2}$ forces a value on $h \uparrow T(i)$. Assume that for each $l,\{|I(s)|: s \in$ $\left.\bigcup_{k \in K\left(I_{2}\right)} \mathcal{S}\left(l, k, f_{2} \cup \chi_{1}\left(I_{2}\right)\right)\right\}$ is unbounded. We could then find an increasing sequence $\left\{k_{l}: l \in \omega\right\} \subset K\left(I_{2}\right)$ and corresponding $s\left(k_{l}\right) \in \mathcal{S}\left(l, k_{l}, f_{2} \cup \chi_{1}\left(I_{2}\right)\right)$ with $\left\{\left|I\left(s\left(k_{l}\right)\right)\right|: l \in \omega\right\}$ diverging, in which case the condition $f_{2} \cup \chi_{1}\left(I_{2}\right) \cup$ $\bigcup_{l} s\left(k_{l}\right)$ would be guilty of forcing a value on $\dot{h} \uparrow T(i)$ for each $i \in \bigcup_{l} I\left(s\left(k_{l}\right)\right)$ $\in \mathfrak{I}^{*}$ - a contradiction.

Therefore there is some $l_{0}$ such that for all $k \in K\left(I_{2}\right)$, and $s \in \mathcal{S}\left(l_{0}, k, f_{2} \cup\right.$ $\left.\chi_{1}\left(I_{2}\right)\right)$, the set $I(s)$ has cardinality less than $l_{0}$. Now choose an increasing sequence $\left\{k_{l}: l \in \omega\right\} \subset K\left(I_{2}\right)$ so that $\left|I_{2} \cap S^{k_{l}}\right|$ has cardinality greater than $l_{0}+2^{2^{l}}$. Choose any condition $f^{\dagger}<f_{2} \cup \chi_{1}\left(I_{2}\right)$ so that $\mathbb{N} \backslash \operatorname{dom}\left(f^{\dagger}\right) \subset \bigcup_{l} S^{k_{l}}$ and $\left|S^{k_{l}} \backslash \operatorname{dom}\left(f^{\dagger}\right)\right|=l$ for all $l$. Notice that $\mathcal{S}\left(l_{0}, k_{l}, f^{\dagger}\right)$ has cardinality at most $2^{2^{l}}$. For each $l$ and $s \in \mathcal{S}\left(l_{0}, k_{l}, f^{\dagger}\right)$, choose an $i_{s} \in I_{2} \cap S^{k_{l}}$ such that $s \cup f^{\dagger}$ does not force a value on $\dot{h}\left\lceil T\left(i_{s}\right)\right.$. Ensure that the selection is such that $i_{s} \neq i_{s^{\prime}}$ for distinct $s, s^{\prime} \in \mathcal{S}\left(l_{0}, k_{l}, f^{\dagger}\right)$. Next, for each $l$ and $s \in S\left(l_{0}, k_{l}, f^{\dagger}\right)$, choose $t_{s} \in T\left(i_{s}\right)$ and distinct $u_{s}, w_{s}$ each with the property that there is some extension of $s \cup f^{\dagger}$ forcing $\dot{h}\left(t_{s}\right)$ to have that value.

We now define an ultrafilter in $\mathbb{N}^{*}$. For each $g$, let $X(g)=\left\{i_{s}: s \in\right.$ $\left.\bigcup_{l} \mathcal{S}\left(l_{0}, k_{l}, g\right)\right\}, U(g)=\left\{u_{s}: i_{s} \in X(g)\right\}$, and $W(g)=\left\{w_{s}: i_{s} \in X(g)\right\}$. Let $z \in \mathbb{N}^{*}$ be any ultrafilter which extends the family $\left\{X(g): g \in \mathfrak{F}, g<f^{\dagger}\right\}$.

Since $U\left(f^{\dagger}\right) \cap W\left(f^{\dagger}\right)$ is empty, there is some $g<f^{\dagger}$ such that either $g \Vdash_{\mathbb{P}}$ " $U\left(f^{\dagger}\right) \notin z$ " or $g \Vdash_{\mathbb{P}}$ "W( $\left.W f^{\dagger}\right) \notin z$ " (by symmetry assume $U\left(f^{\dagger}\right) \notin z$ ). By possibly extending $g$, there is an $X \in x$ such that $g \Vdash_{\mathbb{P}} " F(X) \cap U\left(f^{\dagger}\right)={ }^{*} \emptyset "$. Since $X \cap X(g)$ is infinite we can choose an infinite set $L \subset \mathbb{N}$ such that for each $l \in L, s_{l}=g\left\lceil S^{k_{l}} \in \mathcal{S}\left(l_{0}, k_{l}, g\right)\right.$ and $s_{l} \in X$. For each $l \in L$, let $s_{l}^{*} \in \mathcal{S}_{l}$ be chosen so that $s_{l} \subset s_{l}^{*}$ and $s_{l}^{*} \cup f^{\dagger}$ forces $\dot{h}\left(i_{s_{l}}\right)=u_{s_{l}}$. By genericity of $\mathfrak{F}$, there is a $g^{\dagger}<g$ such that $L^{\prime}=\left\{l \in L: S^{k_{l}} \subset \operatorname{dom}\left(g^{\dagger}\right)\right.$ and $\left.s_{l}^{\dagger} \subset g^{\dagger} \uparrow S^{k_{l}}\right\}$ is infinite. Since

$$
g^{\dagger} \Vdash_{\mathbb{P}} "\left\{u_{s_{l}}\right\}_{l \in L^{\prime}}=\dot{h}\left[\left\{i_{s_{l}}\right\}_{l \in L^{\prime}}\right]={ }^{*} F\left(\left\{i_{s_{l}}\right\}_{l \in L^{\prime}}\right) \subset^{*} \mathbb{N} \backslash U\left(f^{\dagger}\right) ",
$$

we have our contradiction.

This completes the proof of Claim 5 . 


\section{Questions}

Question 6.1. Assume PFA. If $G$ is $\mathbb{P}_{2}$-generic, and $\mathbb{N}^{*}=A \bowtie B$ is the generic tie-point introduced by $\mathbb{P}_{2}$, is it true that $A$ is not homeomorphic to $\mathbb{N}^{*}$ ? Is it true that $\tau(x)=2$ ? Is it true that each tie-point is a symmetric tie-point?

REMARK 1 . The tie-point $x_{3}$ introduced by $\mathbb{P}_{3}$ does not satisfy $\tau\left(x_{3}\right)=3$. This can be seen as follows. For each $f \in \mathbb{P}_{3}$, we can partition $L_{f}$ into $\left\{i \in \operatorname{dom}(f): i<f(i)<f^{2}(i)\right\}$ and $\left\{i \in \operatorname{dom}(f): i<f^{2}(i)<f(i)\right\}$.

It seems then that the tie-points $x_{l}$ introduced by $\mathbb{P}_{l}$ might be better characterized by the property that there is an autohomeomorphism $F_{l}$ of $\mathbb{N}^{*}$ such that fix $\left(F_{l}\right)=\left\{x_{l}\right\}$, and each $y \in \mathbb{N}^{*} \backslash\{x\}$ has an orbit of size $l$.

REMARK 2. A small modification to the poset $\mathbb{P}_{2}$ will result in a tiepoint $\mathbb{N}^{*}=A \underset{x}{\bowtie B}$ such that $A$ (hence the quotient space by the associated involution) is homeomorphic to $\mathbb{N}^{*}$. The modification is to build into the conditions a map from the pairs $\{i, f(i)\}$ into $\mathbb{N}$. A natural way to do this is to set $f \in \mathbb{P}_{2}^{+}$if $f$ is a 2-to- 1 function such that for each $n, f$ maps $\operatorname{dom}(f) \cap\left(2^{n+1} \backslash 2^{n}\right)$ into $2^{n} \backslash 2^{n-1}$, and again $\lim \sup _{n}\left|2^{n+1} \backslash\left(\operatorname{dom}(f) \cup 2^{n}\right)\right|$ $=\infty$. $\mathbb{P}_{2}^{+}$is ordered by almost containment. The generic filter introduces an $\omega_{2}$-sequence $\left\{f_{\alpha}: \alpha \in \omega_{2}\right\}$ and two ultrafilters: $x \supset\left\{\mathbb{N} \backslash \operatorname{dom}\left(f_{\alpha}\right): \alpha \in \omega_{2}\right\}$ and $z \supset\left\{\mathbb{N} \backslash \operatorname{range}\left(f_{\alpha}\right): \alpha \in \omega_{2}\right\}$. For each $\alpha$ and $a_{\alpha}=\left\{i \in \operatorname{dom}\left(f_{\alpha}\right): i=\right.$ $\min \left(f_{\alpha}^{-1}\left(f_{\alpha}(i)\right)\right\}$, we set $A=\{x\} \cup \bigcup_{\alpha} a_{\alpha}^{*}$ and $B=\{x\} \cup \bigcup_{\alpha}\left(\operatorname{dom}\left(f_{\alpha}\right) \backslash a_{\alpha}\right)^{*}$; then $\mathbb{N}^{*}=A \searrow_{x} B$ is a symmetric tie-point. Finally, the map $F: A \rightarrow \mathbb{N}^{*}$ defined by $F(x)=z$ and $F\left\lceil A \backslash\{x\}=\bigcup_{\alpha}\left(f_{\alpha}\right)^{*}\right.$ is a homeomorphism.

Question 6.2. Assume PFA. If $L$ is a finite subset of $\mathbb{N}$ and $\mathbb{P}_{L}=\prod\left\{\mathbb{P}_{l}\right.$ : $l \in L\}$, is it true in $V[G]$ that there is a finite upper bound to $\tau(x)$ for the tie-points $x$; and if $1 \notin L$, then every tie-point is a symmetric tie-point?

Acknowledgements. Research of the first author was supported by NSF grant No. NSF-DMS 20060114. The research of the second author was supported by the United States-Israel Binational Science Foundation (BSF Grant no. 2002323), and by NSF grant No. NSF-DMS 0600940.

\section{References}

[1] J. E. Baumgartner, Applications of the proper forcing axiom, in: Handbook of SetTheoretic Topology, North-Holland, Amsterdam, 1984, 913-959.

[2] A. Dow, Extending real-valued functions in $\beta \kappa$, Fund. Math. 152 (1997), 21-41.

[3] A. Dow and G. Techanie, Two-to-one continuous images of $\mathbb{N}^{*}$, ibid. 186 (2005), 177-192.

[4] R. Levy, The weight of certain images of $\omega^{*}$, Topology Appl. 153 (2006), 2272-2277. 
[5] S. Shelah and J. Steprāns, Non-trivial homeomorphisms of $\beta N \backslash N$ without the Continuum Hypothesis, Fund. Math. 132 (1989), 135-141.

[6] —, -, Somewhere trivial autohomeomorphisms, J. London Math. Soc. (2) 49 (1994), $569-580$.

[7] —, - Martin's axiom is consistent with the existence of nowhere trivial automorphisms, Proc. Amer. Math. Soc. 130 (2002), 2097-2106.

[8] J. Steprāns, The autohomeomorphism group of the Čech-Stone compactification of the integers, Trans. Amer. Math. Soc. 355 (2003), 4223-4240.

[9] B. Veličković, Definable automorphisms of $\mathcal{P}(\omega) /$ fin, Proc. Amer. Math. Soc. 96 (1986), 130-135.

[10] -, OCA and automorphisms of $\mathcal{P}(\omega) /$ fin, Topology Appl. 49 (1993), 1-13.

University of North Carolina at Charlotte

Charlotte, NC 28223, U.S.A.

E-mail: adow@uncc.edu
Department of Mathematics Rutgers University Hill Center Piscataway, NJ 08854-8019, U.S.A. E-mail: shelah@math.rutgers.edu

Current address: Institute of Mathematics Hebrew University Givat Ram, Jerusalem 91904, Israel

Received 28 August 2007;

in revised form 14 August 2008 and 3 January 2009 